\title{
ANOMALOUS COSMIC RAYS
}

\author{
Report of Working Group 3
}

\section{B. KLECKER ${ }^{1}$ and R.A. MEWALDT ${ }^{2}$ \\ Co-Chairs}

J.W. BIEBER ${ }^{3}$, A.C. CUMMINGS ${ }^{2}$, L. DRURY ${ }^{4}$, J. GIACALONE 5 , J.R. JOKIPII ${ }^{5}$, F.C. JONES ${ }^{6}$, M.B. KRAINEV ${ }^{7}$, M.A. LEE ${ }^{8}$, J.A. LE ROUX ${ }^{9}$, R.G. MARSDEN ${ }^{10}$, F.B. MCDONALD ${ }^{9}$, R.B. MCKIBBEN ${ }^{11}$ and C.D. STEENBERG ${ }^{12}$ Participants

M.G. BARING ${ }^{6}$, D.C. ELLISON ${ }^{13}$, L.J. LANZEROTTI ${ }^{14}$, R.A. LESKE $^{2}$, J.E. MAZUR ${ }^{9}$, H. MORAAL ${ }^{12}$, M. OETLIKER ${ }^{1}$, V.S. PTUSKIN ${ }^{15}$, R.S. SELESNICK ${ }^{16}$ and K.J. TRATTNER ${ }^{17}$

Contributing Authors not participating in the Workshop

' Max-Planck-Institut für extraterrestrische Physik, 85740 Garching, Germany

${ }^{2}$ California Institute of Technology, Space Radiation Laboratory,

Downs Lab. M/C 220-47, Pasadena, CA 91125, USA

${ }^{3}$ University of Delaware, Newark, DE 19716, USA

${ }^{4}$ Dublin Institute of Advanced Studies, School of Cosmic Physics, Dublin, Ireland

${ }^{5}$ Lunar and Planetary Laboratory, The University of Arizona, Tucson, AZ 85721, USA

${ }^{6}$ Laboratory for High Energy Astrophysics, NASA/GSFC, Greenbelt, MD 20771, USA

${ }^{7}$ Lebedev Physical Institute, Moscow, Russia

${ }^{8}$ University of New Hampshire, Durham, NH 03824, USA

${ }^{9}$ University of Maryland, College Park, MD 20742, USA

${ }^{10}$ Space Science Dept. of ESA, Postbus 299, 2200 AG Noordwijk, The Netherlands

$1 "$ Enrico Fermi Institute, University of Chicago, Chicago, IL 60637, USA

${ }^{12}$ Space Research Unit, Department of Physics, Potchefstroom University for CHE, I Potchefstroom, South Africa

${ }^{13}$ Department of Physics, North Carolina State University, Raleigh, NC 27695, USA

${ }^{14}$ AT \& T Bell Laboratories, Murray Hill, NJ 07974, USA

${ }^{15}$ IZMIRAN, Troitsk, Moscow District 142092, Russia

${ }^{16}$ The Aerospace Corporation, Los Angeles, CA 90009, USA

${ }^{17}$ Mullard Space Science Laboratory, Holmbury St. Mary, Dorking, Surrey, UK

\begin{abstract}
We review the observed properties of anomalous cosmic rays and the present status of our knowledge of the processes by which they originate. We compiled a comprehensive set of ACR energy spectral data from various spacecraft throughout the heliosphere during the passes of Ulysses over the poles of the Sun and present first results of a detailed modeling effort. In several contributions, we discuss the questions of injection and possible pre-acceleration of pickup ions, summarize new observations on the ionic charge composition, and present new results on the composition of minor ions in ACRs.
\end{abstract}




\title{
1. Introduction
}

\author{
R.A. Mewaldt, B. Klecker, and A.C. Cummings
}

During the twenty five years since the discovery of anomalous cosmic rays (ACR) there has been enormous progress in delineating their observed properties and in deducing the processes by which they originate (see e.g., reviews by Biswas et al., 1993; Klecker, 1995; and Simpson, 1995). As a result of this progress it is now firmly established that the bulk of ACRs result from interstellar neutrals that have been swept into the heliosphere and ionized to become pickup ions (Fisk et al., 1974). These pickup ions are then convected into the outer heliosphere, where they are accelerated to energies of $\sim 1$ to $100 \mathrm{MeV} / \mathrm{nuc}$, presumably at the solar wind termination shock (Pesses et al., 1981). The ACR component is now generally agreed to include the elements $\mathrm{H}, \mathrm{He}, \mathrm{C}, \mathrm{N}, \mathrm{O}, \mathrm{Ne}$, and Ar.

A number of observations have now verified key aspects of this basic picture. The composition of solar wind pickup ions has been measured directly (e.g. Geiss et al., 1994, and references therein), and neutral interstellar He was observed streaming into the heliosphere (Witte et al., 1993). It has also been demonstrated that the bulk of ACRs are singly-charged (Adams et al., 1991; Klecker et al., 1995), which distinguishes them from other particle components such as solar energetic particles (SEP) and galactic cosmic rays (GCR).

During the past several years of solar minimum conditions several new observations have intensified interest in ACRs, and raised a number of new questions about their origin. In the outer heliosphere the spectra of ACR He and $\mathrm{O}$ are unfolding with time and/or distance and the evolving spectral shapes have been used to estimate the location and strength of the termination shock (Cummings and Stone, 1996). Observations by SAMPEX have shown that at energies $>20 \mathrm{MeV} / \mathrm{nuc}$ most ACRs are no longer singly charged as at lower energies $(<16 \mathrm{MeV} / \mathrm{nuc})$; rather, they have charge states of $q=+2,+3$ and higher (Mewaldt et al., 1996b), which has implications for the nature of the acceleration process (Jokipii, 1996). In addition, Ulysses data have shown that there are apparently additional sources of pickup ions, at least in the inner heliosphere (Geiss et al., 1995), and there is evidence from Wind and Geotail for an enhancement in the low-energy sulfur spectrum similar to that of ACR species (Reames et al., 1996; Takashima et al., 1997).

At the same time, the spatial and temporal variations of ACRs continue to provide new tests of theories for particle transport in the interplanetary medium. The interplanetary flux of ACRs near 1 AU has been found to vary by a factor of $>100$ over the solar cycle (see Figure 1), while multi-spacecraft studies of ACRs by Voyager $1 \& 2$, Pioneer 10 \& 11, Ulysses, SAMPEX, and Wind have demonstrated that the latitudinal gradients of ACRs during the 1990's are indeed positive (Cummings et al., 1995; Trattner et al., 1996), as predicted, but also smaller in magnitude than expected. The status of many of these new aspects of ACR studies has been summarized in the review by Cummings and Stone (1998). 


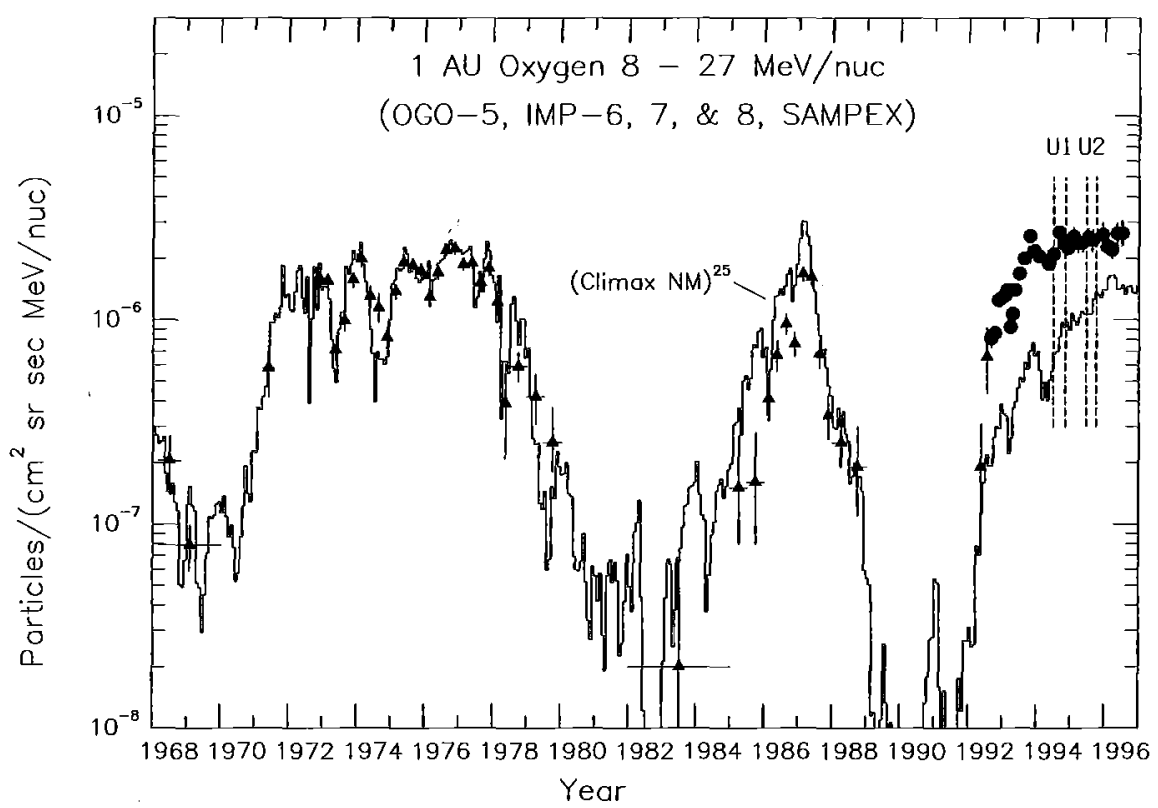

Figure 1. Quiet-time measurements of the flux of 8 to $27 \mathrm{MeV} / \mathrm{nuc}$ oxygen at 1 AU over the past 28 years. Data from prior to 1972 are from OGO-5 and IMP-6; those from 1972 to mid- 1992 are from IMP 7 and 8, after which they are from SAMPEX (see Mewaldt et al., 1993). The solid curve is proportional to the Climax Neutron Monitor counting rate (R. Pyle and J. Simpson, private communication) taken to the 25th power. Note that the flux of ACR oxygen at 1 AU had reached a plateau during the two selected periods (U1 $=1994: 178-309$ and $U 2=95: 170-273$ ) when Ulysses reached maximum heliographic latitude.

The September 1996 and March 1997 meetings of the ACR Working Group at ISSI provided an opportunity to assess recent progress in ACR studies, and to identify new issues that have been raised by this progress. Table 1 presents a summary of the key questions that were identified by the ACR Working Group in September, 1996. At this meeting plans were also outlined for a number of studies that would lead to progress on most if not all of these questions. The initial results of these studies are presented in this chapter.

It was agreed that an important focus of the ACR working group would be to collect and then model data from the various interplanetary spacecraft that were in operation during 1994 and 1995 when Ulysses passed over the poles of the Sun, since these represent unique opportunities to explore the latitudinal distribution of ACRs in the heliosphere under solar minimum conditions. These two periods are identified in the long term ACR record shown in Figure 1. Coincidentally, they also include some of the last data that is available from Pioneer 10. The ACR data set from these periods (including contributions from Voyager 1 and 2, Pioneer 10, Ulysses, SAMPEX, and Wind) is presented in Section 2. The first of what will undoubtedly be several attempts to model these data is presented in Section 3. 
Table I

Top ten questions in anomalous cosmic ray studies.

1) In addition to $\mathrm{H}, \mathrm{He}, \mathrm{C}, \mathrm{N}, \mathrm{O}, \mathrm{Ne}$ and $\mathrm{Ar}$, are there other $\mathrm{ACR}$ species originating in the neutral interstellar medium?

2) Are there ions (or electrons) of other origin (e.g. solar, interplanetary) accelerated at the termination shock?

3) How are pickup ions injected into the (quasi-perpendicular) termination shock?

4) What causes the under-abundance of ACR hydrogen and the apparent mass-dependent acceleration efficiency?

5) Are pickup ions pre-accelerated by interplanetary shocks prior to arriving at the termination shock?

6) What are the implications of ACR spectra and gradients for interplanetary diffusion coefficients and other transport parameters?

7) What can multiply-charged ACRs tell us about the location and time scale of ACR acceleration?

8) How do multiply-charged ACRs affect the interpretation of observed ACR energy spectra?

9) What can ACRs tell us about the location and strength of the termination shock, and about ACR spectra at the shock?

10) Can a self-consistent acceleration/transport model explain observed ACR spectra, gradients, and time variations, as well as those of GCRs?

The so-called "injection problem" was identified as a second important focus of the Working Group. New theoretical ideas are needed to explain how ACRs are injected into the acceleration process, and why hydrogen, especially, but also other light elements are apparently less abundant in ACRs than in pickup ions (see Cummings and Stone, 1996, and references therein). Four of the contributions to this chapter discuss possible approaches to ACR injection, including pre-acceleration in interplanetary space (Sections 6 and 7), and injection and acceleration at the termination shock (Sections 4 and 5).

Recent observations have shown that the ionic charge composition of ACR oxygen is more complex than previously realized, suggesting a new probe of ACR acceleration processes. The new results from SAMPEX in Section 8 show that ACR $\mathrm{N}$ and $\mathrm{Ne}$ are also predominantly multiply-charged at energies $>20 \mathrm{MeV} / \mathrm{nuc}$. Section 7 considers the production of multiply-charged oxygen during interplanetary pre-acceleration of pickup ions, while Section 3 illustrates the possible effect that $\mathrm{He}^{++}$may have on the ACR He spectrum.

The recent evidence for an enhanced flux of pickup $\mathrm{C}$ in the inner heliosphere (Geiss et al., 1995) and for an enhancement in the 3 to $20 \mathrm{MeV} /$ nuc spectrum of sulfur at $1 \mathrm{AU}$ has stimulated new interest in whether there are other species of ACRs from the interstellar neutral source or from other sources such as interplanetary dust. Section 9 presents preliminary results of a search for additional ACR species. 
During the 1970s, following the discovery of ACRs, there was a flurry of experimental and theoretical activity that led quite rapidly to a consensus on the origin of ACRs - a consensus that called out for experimental verification. During the 1980 s there was continuing theoretical progress, but the pace of new experimental results to test these theories was noticeably slowed. The dawn of the current solar minimum in 1992 found the Voyager and Pioneer spacecraft exploring the outer heliosphere at $50 \mathrm{AU}$ and beyond, Ulysses approaching the south pole of the Sun, and a new generation of instruments about to be launched on SAMPEX, Geotail and Wind. The result has been an explosion of new experimental data on pickup ions and anomalous cosmic rays that have once again presented a host of new theoretical challenges and raised new questions to be addressed experimentally. This chapter represents the first results of studies that were initiated to meet these challenges. These approaches appear to be promising, but it is clear that additional work is called for, in part because of the great deal of experimental data that must now be addressed by any successful theory of ACR acceleration and transport. In any case, it appears that over the next few years these studies will continue to offer new insight into a surprisingly broad spectrum of interstellar and heliospheric processes that comprise the ACR phenomenon.

\section{Anomalous Cosmic Ray Data Sets}

R.G. Marsden, A.C. Cummings, B. Klecker, L.J. Lanzerotti, J.E. Mazur, R.B. McKibben, R.A. Mewaldt and K.J. Trattner

\subsection{INTRODUCTION}

The current solar minimum offers a unique opportunity for ACR studies, with an unprecedented array of instruments being carried by spacecraft at widely separated locations in the heliosphere. Of particular importance in this respect were the polar passes of Ulysses in 1994 and 1995, which provided the first mapping of ACR fluxes over the full range of heliolatitudes within $5 \mathrm{AU}$ of the Sun. In order to exploit this opportunity to the full, comprehensive sets of ACR energy spectral data covering the epochs of the two Ulysses polar passes have been assembled with a view to providing theorists with detailed observational constraints to models of ACR acceleration and transport.

\subsection{The DaTa SeTS}

The two time periods chosen over which to average the energy spectral data correspond to the times at which Ulysses was above $70^{\circ}$ heliographic latitude in either heliospheric hemisphere, namely 1994/178.00-309.00 (27 June - 04 November incl.) and 1995/170.00-273.00 (19 June - 29 September incl.). In some cases, the 
Table II

ACR Data Sets Included in the ISSI Data Base.

\begin{tabular}{|c|c|c|c|c|}
\hline \multirow[t]{2}{*}{ Spacecraft } & \multicolumn{2}{|c|}{$\begin{array}{c}\text { S/C Position } \\
\text { (helioc. range/heliog. lat.) }\end{array}$} & \multirow[t]{2}{*}{ Instrument } & \multirow[t]{2}{*}{ ACR Species } \\
\hline & 1994/178-309 & $1995 / 170-273$ & & \\
\hline SAMPEX & \multicolumn{2}{|c|}{ Earth orbit } & $\begin{array}{l}\text { HILT }^{a} \\
\text { LICA }^{b} \\
\text { MAST }\end{array}$ & $\begin{array}{l}\mathrm{O}, \mathrm{Ne}, \mathrm{Ar} \\
\mathrm{O} \\
\mathrm{C}, \mathrm{N}, \mathrm{O}, \mathrm{Ne}\end{array}$ \\
\hline WIND & \multirow{2}{*}{\multicolumn{2}{|c|}{$\begin{array}{c}\text { Near Earth orbit } \\
\text { Earth orbit }\end{array}$}} & LEMT & $\mathrm{He}, \mathrm{O}$ \\
\hline Geotail & & & $\mathrm{HEP}^{c}$ & $\mathrm{C}, \mathrm{N}, \mathrm{O}$ \\
\hline Ulysses & $\begin{array}{l}2.8-1.9 \mathrm{AU} / \\
70-80.2-70 \mathrm{~S}\end{array}$ & $\begin{array}{c}1.7-2.5 \mathrm{AU} / \\
70-80.2-70 \mathrm{~N}\end{array}$ & $\begin{array}{l}\text { COSPIN/LET } \\
\text { HISCALE }\end{array}$ & $\begin{array}{l}\mathrm{C}, \mathrm{N}, \mathrm{O}, \mathrm{Ne} \\
\mathrm{C}, \mathrm{N}, \mathrm{O}, \mathrm{Ne}\end{array}$ \\
\hline Voyager 1 & $56.9 \mathrm{AU} / 32.6 \mathrm{~N}$ & $60.3 \mathrm{AU} / 32.8 \mathrm{~N}$ & CRS & $\mathrm{H}, \mathrm{He}, \mathrm{C}, \mathrm{N}, \mathrm{O}$ \\
\hline Voyager 2 & $43.8 \mathrm{AU} / 12.3 \mathrm{~S}$ & $46.5 \mathrm{AU} / 14.3 \mathrm{~S}$ & CRS & $\mathrm{H}, \mathrm{He}, \mathrm{C}, \mathrm{N}, \mathrm{O}$ \\
\hline Pioneer 10 & $60.4 \mathrm{AU} / 3.2 \mathrm{~N}$ & $62.9 \mathrm{AU} / 3.1 \mathrm{~N}$ & GSFC & $\mathrm{He}, \mathrm{O}$ \\
\hline
\end{tabular}

a Averaging period 1: 1994/201-309 (16.9 d total); period 2: 1995/170-212 (7.9 d)

b Averaging period 1: 1994/208-309 (47.0 d total); period 2: 1995/170-273 (9.6 d)

$c$ No data for 1995. Only quiet times used (38\% of 1994 interval)

data for certain days within these periods have been removed in order to exclude non-quiet times. A summary of the various instruments contributing to, and the ACR species included in, the data sets is presented in Table 2.2.

For ease of manipulation, the data from each instrument, ACR species and period have been assembled in the form of ASCII files, each containing the following information: minimum, maximum and mean energy for each spectral point (in $\mathrm{MeV} / \mathrm{nuc}$ ), the corresponding ACR flux (in units of particles $/\left(\mathrm{cm}^{2} \mathrm{sr} \mathrm{s} \mathrm{MeV/nuc)}\right.$ and corrected for GCR contributions where applicable and/or possible), and its uncertainty. Information concerning the location of the various spacecraft at the time of measurement, also included in the files, is reproduced in Table 2.2.

\subsubsection{Accessing the Data Sets}

The ASCII files containing the spectral data can be accessed via anonymous ftp on the ISSI server vega . unibe. ch. A README. TXT file is included in the subdirectory / workshop/cosmrays / acrdat that provides additional information concerning the data sets.

N.B. The primary purpose of the data sets is to assist in ACR modeling. Authors wishing to use a given data set for publication purposes are strongly urged to contact the PI of the instrument in question prior to submission. 
$1 \mathrm{AU}$
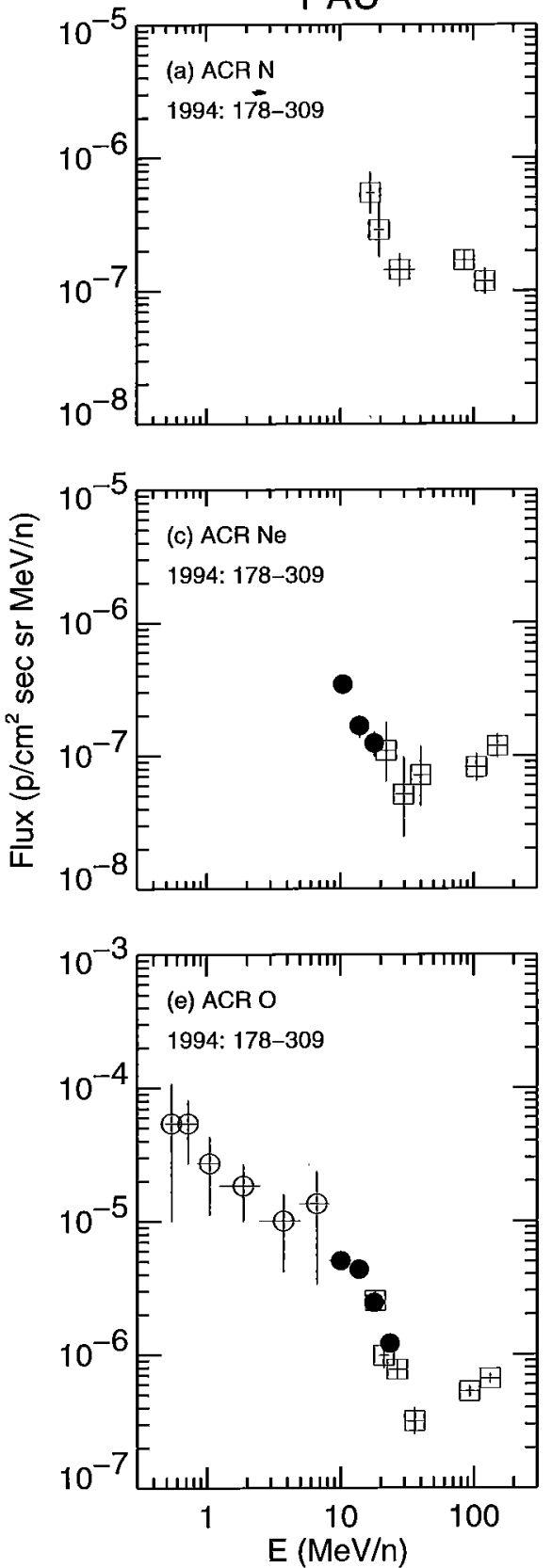

$1 \mathrm{AU}$
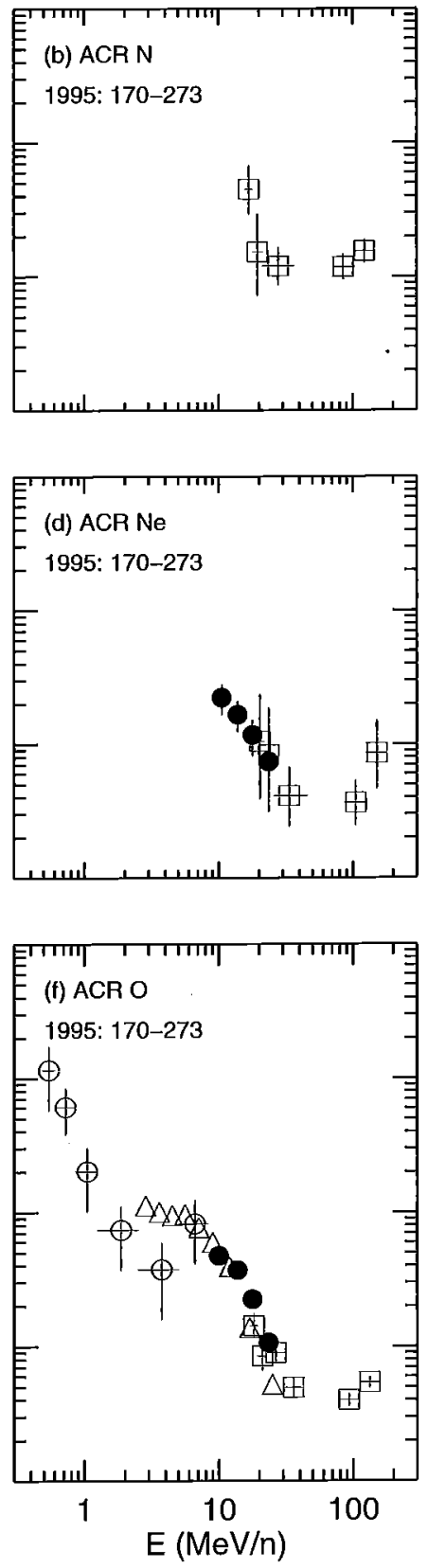

Figure 2. ACR Nitrogen $(\mathrm{N})$, Neon $(\mathrm{Ne})$ and Oxygen $(\mathrm{O})$ spectra for the two ISSI periods measured at 1 AU by SAMPEX/LICA (open circles), SAMPEX/HILT (filled circles), SAMPEX/MAST (open squares), and Wind/LEMT (open triangles). The low energy ( $<3 \mathrm{MeV} / \mathrm{nuc}$ ) spectra include a solar or interplanetary component. Note that for MAST data GCR contributions are not subtracted and dominate above $\sim 40 \mathrm{MeV} / \mathrm{nuc}$. 


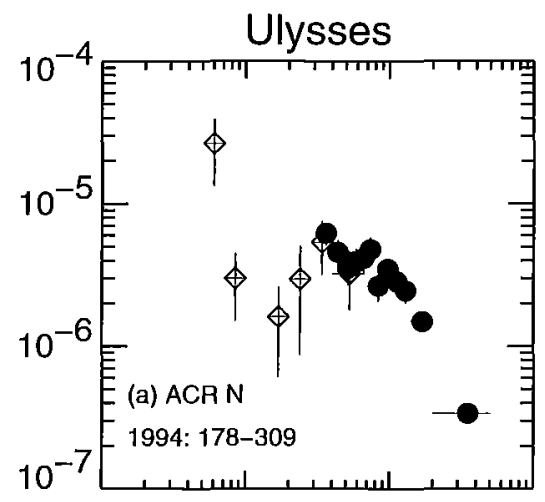

Ulysses
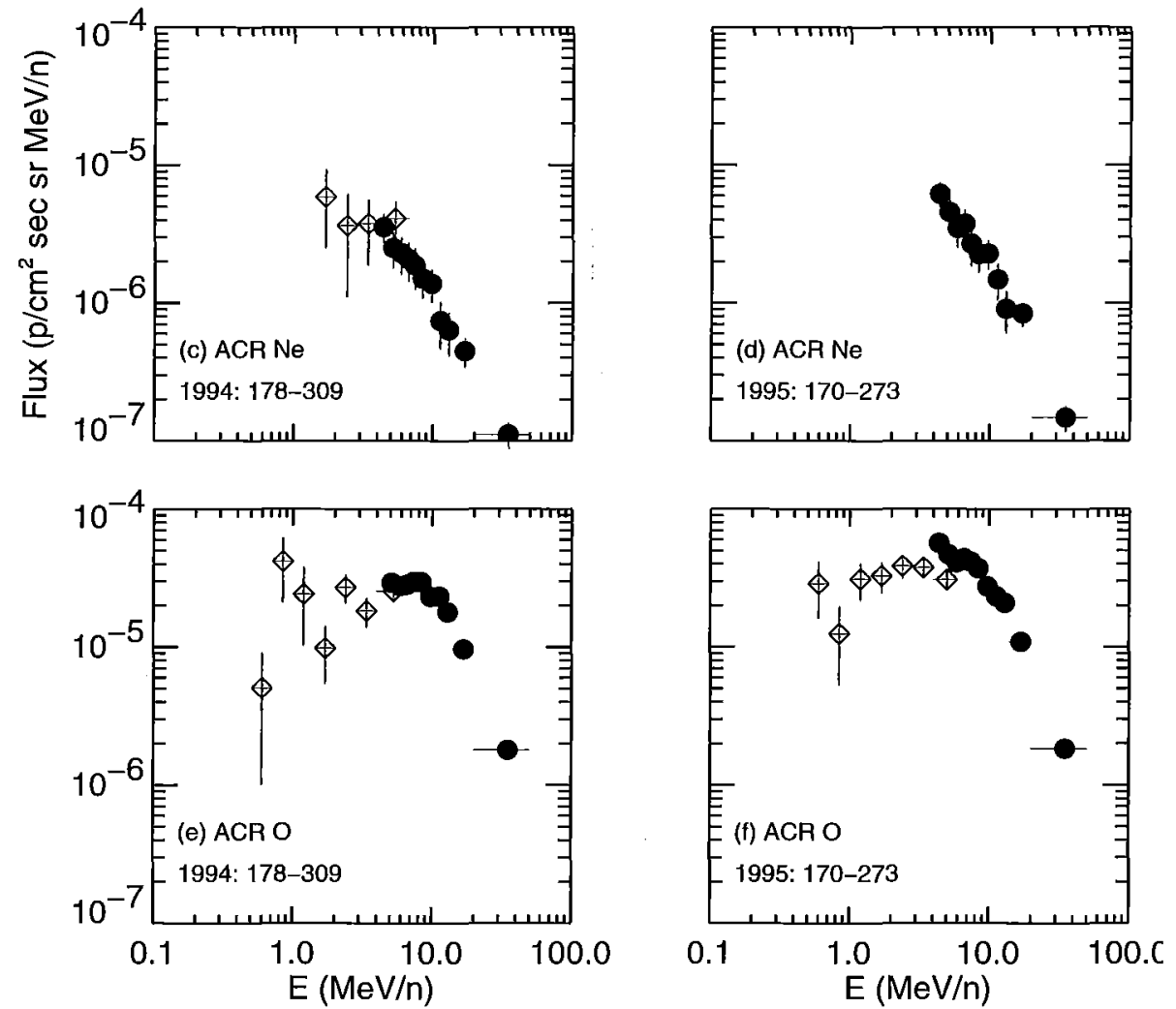

Figure 3. ACR Nitrogen $(\mathrm{N})$, Neon (Ne) and Oxygen $(\mathrm{O})$ spectra for the two ISSI periods measured at Ulysses by COSPIN/LET (filled circles) and HI-SCALE (open diamonds).

\subsection{THE ENERGY SPECTRA}

Sample energy spectra from the ISSI data base are plotted in Figures 2.2 to 2.2. Many more spectra are contained in the data base than can be displayed in the restricted space available here. For comparison purposes, the spectra have been 

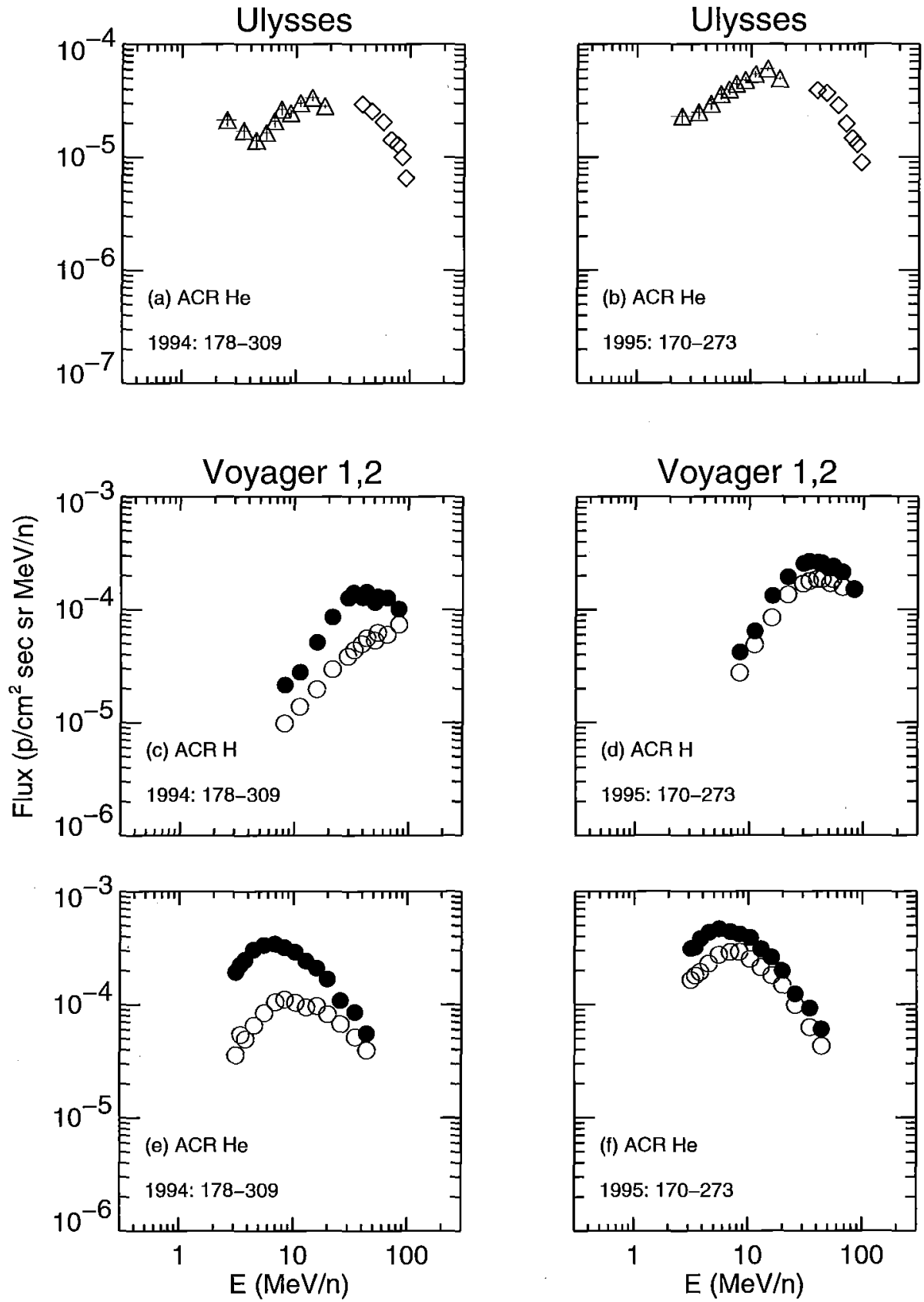

Figure 4. ACR Hydrogen $(\mathrm{H})$ and Helium (He) spectra for the two ISSI periods measured at Ulysses (upper panels) and in the outer heliosphere at Voyager 1 and 2 (center and lower panels; V1: filled circles, V2: open circles). The Ulysses data are from the COSPIN/LET (open triangles) and COSPIN/HET (open diamonds, from McKibben et al., 1996). 

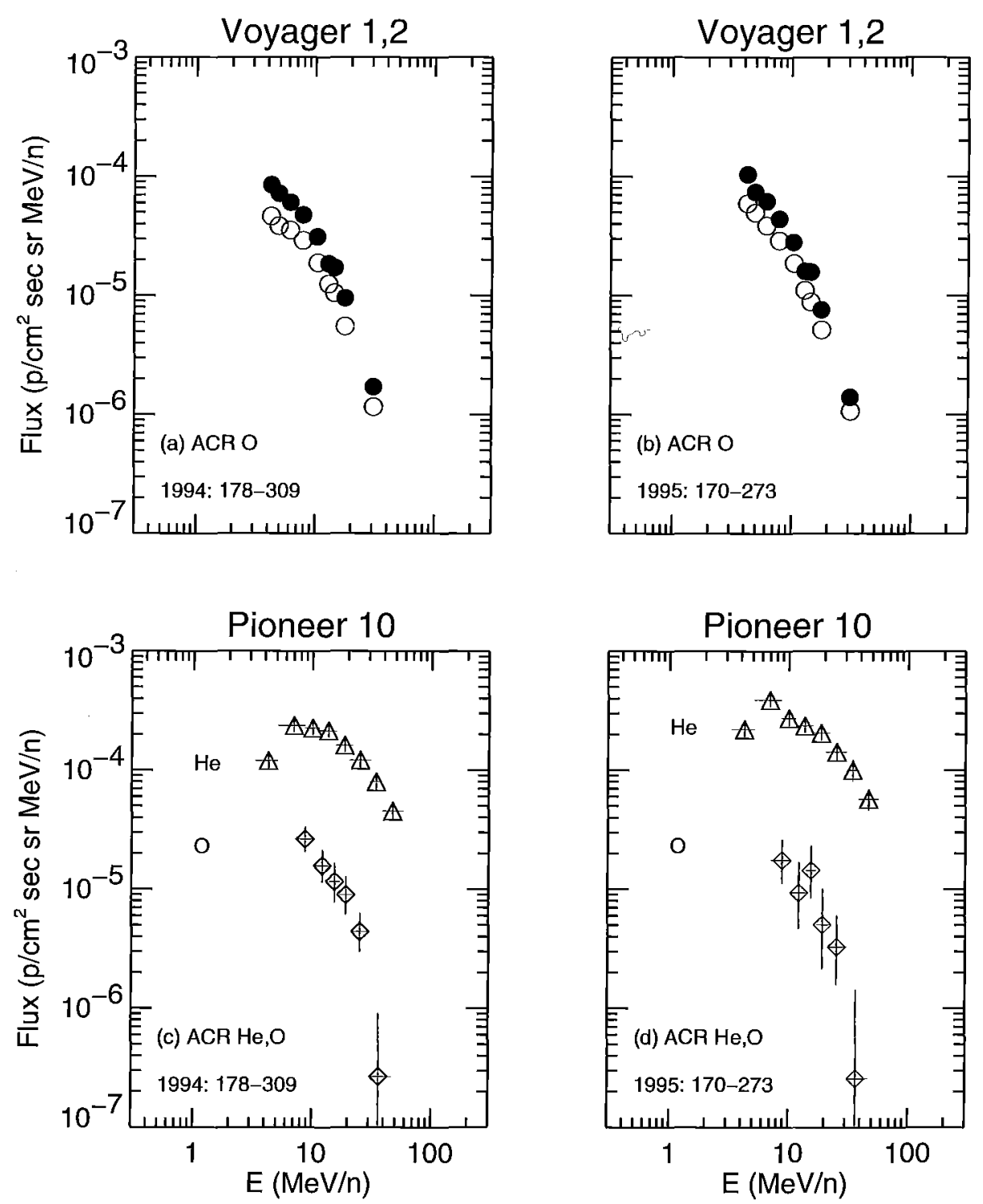

Figure 5. ACR Oxygen (O) and Helium (He) spectra for the two ISSI periods measured in the outer heliosphere at Voyager 1 and 2 (upper panels; V1: filled circles, V2: open circles), and at Pioneer 10 (lower panels).

grouped according to heliospheric location of the spacecraft from which the measurements were taken (i.e., SAMPEX, Wind, Geotail and IMP-8 at 1 AU, Ulysses at high latitudes, and Voyager 1/2 and Pioneer 10 in the outer heliosphere). It should be noted that no inter-normalization has taken place; nevertheless, the spectra from the different instruments and/or spacecraft for a given location show generally good agreement in areas where the energy ranges overlap. In cases where flux 
values are plotted without error bars, these are smaller than the symbols used. Note also that the low energy spectra $(\mathrm{E} \leq 3 \mathrm{MeV} / \mathrm{nuc})$ are not corrected for a solar or interplanetary component. As was recently shown by Tappin and Simnett (1996), contributions by e.g. CIRs can be substantial in this energy range and need to be corrected accordingly in order to obtain ACR spectra at low energies (see also Simnett and Roelof, 1998). References to the papers describing the instruments, and to additional published spectra are also contained in the archive.

\section{Modulation of the Anomalous and Galactic Components of Cosmic Ray $\mathbf{H}$ and He as described by a Full-Drift Two-Dimensional Acceleration Model}

C.D. Steenberg, H. Moraal and F.B. McDonald

\subsection{INTRODUCTION}

The deep-space missions of Pioneers 10 and 11 and Voyagers 1 and 2 have contributed greatly to our understanding of the modulation of galactic and anomalous cosmic rays in the outer heliosphere. The Space Research Unit of the Potchefstroom University has focused primarily on developing numerical models to solve the cosmic ray transport equation, as discussed at length by Reinecke et al. (1993). The availability of new outer heliospheric observations, made during a $q A>0$ solar cycle, provides new opportunities to study the modulation of cosmic rays in the heliosphere.

Our present studies are a continuation of the work by McDonald et al. (1992), Reinecke et al. (1993) and Reinecke et al. (1996), that applied a stationary twodimensional modulation model (excluding shock acceleration) to the 1977/78 and 1987 GCR Proton, GCR and ACR He, and ACR O, as well as of Steenkamp and Moraal (1993), Steenkamp (1995), Steenberg and Moraal (1996), and Steenberg and Moraal (1997) that applied a time-dependent two-dimensional shock acceleration model (Steenkamp, 1995) to these sets of observations. We now apply the latter model to $\mathrm{H}$ and $\mathrm{He}$ observations made during the first and second Ulysses polar passes in 1994/95, as well as recent 1996 observations, fitting both the ACR and GCR components simultaneously, a method which places new limits on the scattering parameters in the model.

\subsection{THE MODEL}

Steenkamp (1995) developed a numerical code that solves the time-dependent cosmic ray transport equation

$$
\frac{\partial f}{\partial t}=\nabla \cdot(\mathbf{K} \cdot \nabla f-f \boldsymbol{V}) \frac{1}{3 p^{2}}(\nabla \cdot \boldsymbol{V}) \frac{\partial}{\partial p}\left(p^{3} f\right)+Q,
$$

in a two-dimensional axisymmetric heliosphere for the omnidirectional distribution function $f(r, \theta, P)$, as a function of radial distance, $r$, polar angle, $\theta$, and rigidity 
$P=p c / q$, with $p$ the particle momentum, $c$ the speed of light and $q$ its ionic charge. In our calculations we placed the modulation boundary at $r_{b}=120 \mathrm{AU}$, and a compression ratio $s=4$ solar wind termination shock (SWTS) at $r_{s}=90$ AU. We used a nonlinear radial grid with 100 intervals inside the SWTS and 40 from the SWTS to the outer boundary, with the smallest spacing of 0.04 AU around the SWTS. At the boundary the intensity was set equal to the assumed local interstellar spectrum,

$$
j_{b}=P^{2} f_{b}=N \beta /\left(T+0.5 E_{0}\right)^{2.6} \text { particles } /\left(\mathrm{m}^{2} \mathrm{sr} \mathrm{s} \mathrm{MeV/nuc}\right)
$$

for both the GCR $\mathrm{H}$ and He calculations, where $P$ is particle rigidity and $T$ is kinetic energy per nucleon in units of $\mathrm{MeV} /$ nuc. The factor $N$ is a normalization constant, $\beta$ denotes the particle speed relative to that of light, and $E_{0}$ is the rest mass energy of a proton. For the ACR component, particles were injected at the SWTS using the source function

$$
Q(r, P)=Q_{0} \delta\left(r-r_{s}\right) \delta\left(P-P_{i}\right),
$$

with an injection rigidity of $P_{i}=p c / q=0.1 \mathrm{GV}\left(T_{i} \approx 5 \mathrm{MeV}\right.$ for protons, $T_{i} \approx 0.3 \mathrm{MeV} /$ nuc for $\mathrm{He}^{+}, T_{i} \approx 1.25 \mathrm{MeV} /$ nuc for $\mathrm{He}^{++}$). The value of $Q_{0}$ is determined by the ionization efficiency of interstellar neutrals and the injection efficiency of thermal particles at the shock, and was taken to be a free parameter. At the SWTS the radial solar wind speed, $V$, discontinuously drops by the compression ratio, $s=4$, from $V=V_{0} l(\theta)$ to $V=V_{0} l(\theta)\left(r_{s} / r\right)^{2} / s \mathrm{~km} / \mathrm{s}$ outside the shock, with $V_{0}=400 \mathrm{~km} / \mathrm{s}$ and $l(\theta)=1.5+0.5 \cos (2 \theta)$ increasing from 1 in the ecliptic $(\theta=\pi / 2)$ to 2 over the poles $(\theta=0, \pi))$, as shown in panel A of Figure 11. A linear latitudinal grid, with 30 intervals of $3^{\circ}$ each, running from the pole $\left(\theta=0^{\circ}\right)$ to the ecliptic $(\theta=\pi / 2)$ was used, assuming latitudinal symmetry around the solar poles and the ecliptic, i.e. $\partial f / \partial \theta=0$ for $\theta=0, \pi / 2$. A rigidity grid from $P=0.09$ to $20 \mathrm{GV}$, divided logarithmically in 264 intervals, was used, with the boundary conditions that the intensity is zero at the lowest rigidity, and that no modulation takes place at the highest. All solutions are time-asymptotic for a total model time of 2.6 years.

Also included are the effects of particle drifts in an unmodified Parker spiral field, with a field strength of $B=5 \mathrm{nT}$ at Earth and a simulated neutral sheet with a tilt angle of $10^{\circ}$. These magnetic field parameters were not varied between the different observation periods, because drift effects only had a minimal effect on the model calculations due to the large magnitude of the diffusion coefficients used.

\subsection{Data Base}

We apply the model described above to $\mathrm{H}$ and $\mathrm{He}$ observations made during the $q A>0$ drift cycle for the periods of the first and second Ulysses polar passes (1994/178-309 and 1995/170-273 respectively) as well as during 1996. Table III shows the sources of the data, as well as the observation periods, if different from 
Table III

Summary of data collection periods and sources. The abbreviation ISSI indicates that the data are from the source mentioned in Section 2, while GSFC indicates data from Goddard Space Flight Center experiments. The observation periods are 1994/178-309 and 1995/170-273, unless otherwise indicated by the numbers in brackets for a particular year.

\begin{tabular}{llllllll}
\hline & \multicolumn{3}{c}{$\mathbf{H}$} & \multicolumn{3}{c}{ He } \\
Spacecraft & 1994 & \multirow{2}{*}{1995} & 1996 & 1994 & 1995 & 1996 \\
\hline IMP8 & & & GSFC (1-131) & & & GSFC (1-131) \\
V1 & ISSI & ISSI & GSFC (1-153) & ISSI & ISSI & GSFC (1-153) \\
V2 & ISSI & ISSI & GSFC (1-32) & ISSI & ISSI & GSFC (1-32) \\
P10 & GSFC & GSFC & GSFC (1-138) & ISSI & ISSI & GSFC (1-138) \\
& & $(169-247)$ & & & & & \\
\hline
\end{tabular}

those mentioned above. In this table, sets marked "ISSI" are more fully described in Section 2, while those marked "GSFC" are from Goddard Space Flight Center experiments. These observations are illustrated in Figure 3.3, which serves as a prototype for presenting the observations in this contribution.

The observed $1996 \mathrm{H}$ and He spectra cover a broad energy band from 3$300 \mathrm{MeV}$ for $\mathrm{H}$ and 3-500 MeV/nuc for He, clearly showing both the ACR and GCR components of these species. Furthermore, the spectral peak energies of both these components are included in these energy ranges, enabling us to restrict our parameter choice to a very narrow range of values. In contrast, the 1994 and 1995 data did not include the GCR component.

\subsection{Model Calculations}

We now present results of our first attempt to model the ACR and GCR observations presented above, using a time-dependent acceleration/re-acceleration and modulation model. Following the success in fitting 1987 ACR He and O spectra by Steenberg and Moraal (1997), we used the simplest possible spatial diffusion model, namely spatially independent radial and latitudinal diffusion coefficients, $\kappa_{r r}=\kappa_{r r 0} \beta P$ and $\kappa_{\theta \theta}=\kappa_{\theta \theta 0} \beta P$, illustrated in Figure 7 as a first step. The differences in the curves shown are accounted for by the different values of $\beta$, at the same rigidity, which are determined by the particle mass number to charge ratio, $A / Z$.

According to Le Roux et al. (1996), the magnitude of $\kappa_{r r}$ at the shock determines the curvature cutoff of the spectra at the shock. Using our set of parameters, the form of these shock spectra turns out to be one of the important modulation parameters that determines the characteristics of modulated ACR spectra. Thus, our choice of the magnitude of $\kappa_{\tau r}$ at the shock was restricted by the form of the shock spectra, that, along with other modulation parameters, determines the form of the modulated ACR spectra. The model results for ACR and GCR $\mathrm{H}$ and $\mathrm{He}$ are discussed in the next section. 


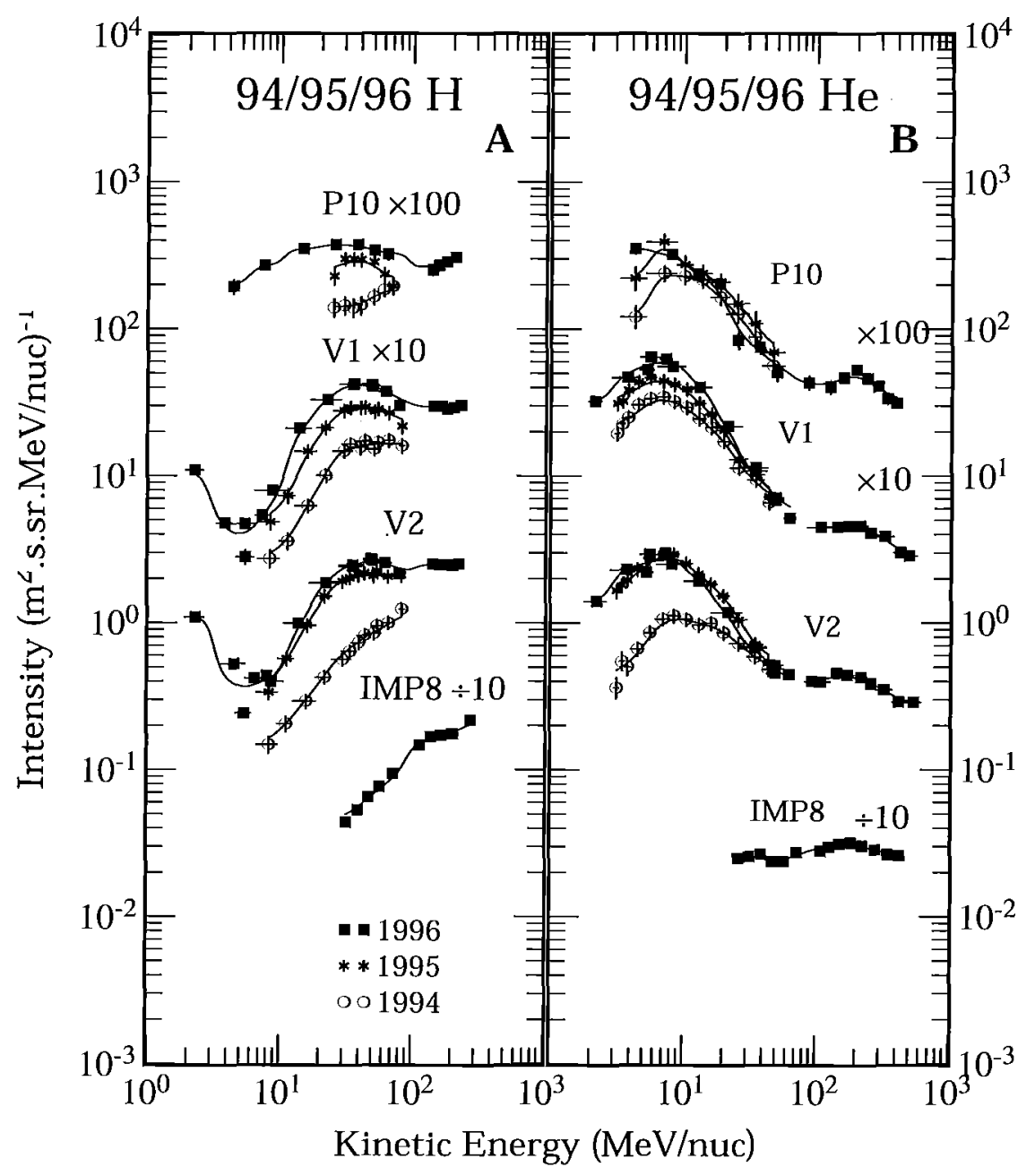

Figure 6. Observed spectral intensities during 1994, 1995 and 1996 for $\mathrm{H}$ (panel A) and He (panel B). In this Figure the data sets from different spacecraft are separated by factors of ten to aid visualization, while a solid line was drawn through every set of points to guide the eye.

\subsubsection{Protons}

ACR and GCR H spectra were calculated for each spacecraft position. These two calculated spectra were added to give the total calculated $\mathrm{H}$ intensity.

Figure 8 shows fits to the 1994, 1995 and $1996 \mathrm{H}$ spectra, obtained using the parameters as summarized in Table IV. Note that the values of $N$ in this table are such that the GCR input spectrum in (2) is about three times lower than the standard value, used by, e.g., Steenberg and Moraal (1996). This was needed because of the large magnitude of $\kappa_{r r}$ (required to fit the ACR spectra). Careful comparison of 


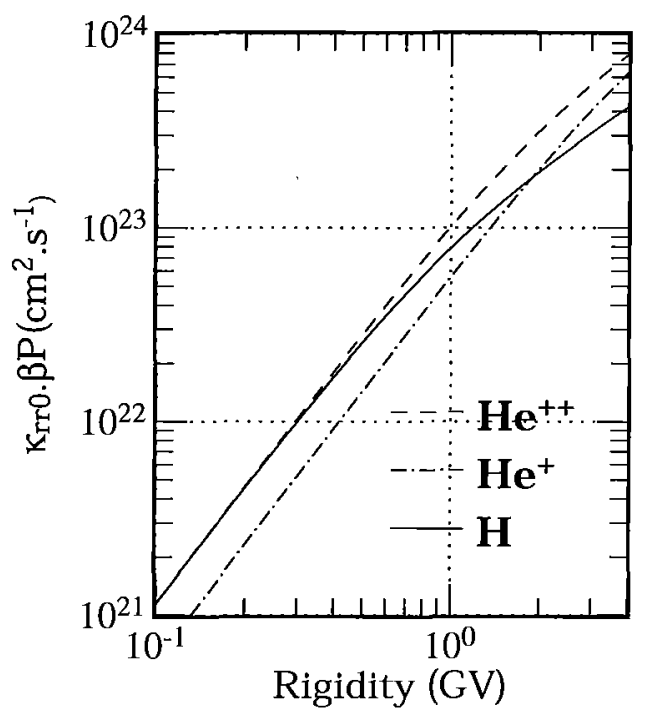

Figure 7. Spatially independent radial diffusion coefficients used to fit the observations as a function of rigidity. The different forms are due to the species dependence of $\beta$ at a given rigidity.

Table IV

Summary of the parameters used to fit the spectra observations in 1994, 1995, and 1996. The diffusion coefficients are in units of $\mathrm{cm}^{2} / \mathrm{s}\left(\times 10^{23}\right), Q_{0}$ in particles $/\left(\mathrm{m}^{2} \mathrm{~s}\right)\left(\times 10^{-23}\right)$, while $N$ is in particles $(\mathrm{MeV} / \mathrm{nuc})^{1.6} /\left(\mathrm{m}^{2} \mathrm{~s} \mathrm{sr}\right)$

\begin{tabular}{|c|c|c|c|c|c|c|c|c|c|}
\hline & \multicolumn{4}{|c|}{$\mathbf{H}$} & \multicolumn{5}{|c|}{ - He } \\
\hline & $\kappa_{r r 0}$ & $\kappa_{\theta \theta 0}$ & $Q_{0}$ & $N$ & $\kappa_{r r 0}$ & $\kappa_{\theta \theta 0}$ & $Q_{0}\left(\mathrm{He}^{+}\right)$ & $Q_{0}\left(\mathrm{He}^{++}\right)$ & $N$ \\
\hline 1994 & 1.08 & 1.08 & 3.71 & 3.82 & 2.16 & 2.16 & 4.74 & 4.74 & 0.319 \\
\hline 1995 & 1.08 & 1.08 & 7.41 & 3.82 & 2.16 & 2.16 & 9.19 & 4.74 & 0.319 \\
\hline 1996 & 1.08 & 1.08 & 7.41 & 3.82 & 2.16 & 2.16 & 9.19 & 4.74 & 0.319 \\
\hline
\end{tabular}

the calculated and observed spectra shows that the calculated ACR gradients are somewhat larger than the observed ones.

To evaluate the diffusion coefficients used in this study, we first note that for

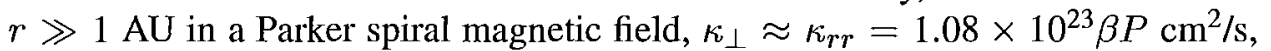
which is $\approx 3.9$ times the value of $\kappa_{\perp}=3.6 \times 10^{22} \beta P$ used by Steenberg and Moraal (1997) to fit the $1997 \mathrm{H}$ observations.

Table $\mathrm{V}$ shows the energies at which the maximum ACR $\mathrm{H}$ contribution to the total intensity occurs for the calculated spectra. For $1 \mathrm{AU}$ these maximum contributions of about $15 \%$ occur at $5.3 \mathrm{MeV}$, while they occur at $23 \mathrm{MeV}$ for all the spacecraft in the outer heliosphere. The relative size of the ACR contribution increases toward the outer heliosphere, as well as from 1994 to 1996, with a maximum calculated contribution of $89 \%$ at Voyager 1 in 1996.

Figure 9 shows radial intensity profiles for the ACR component. Panel A clearly 


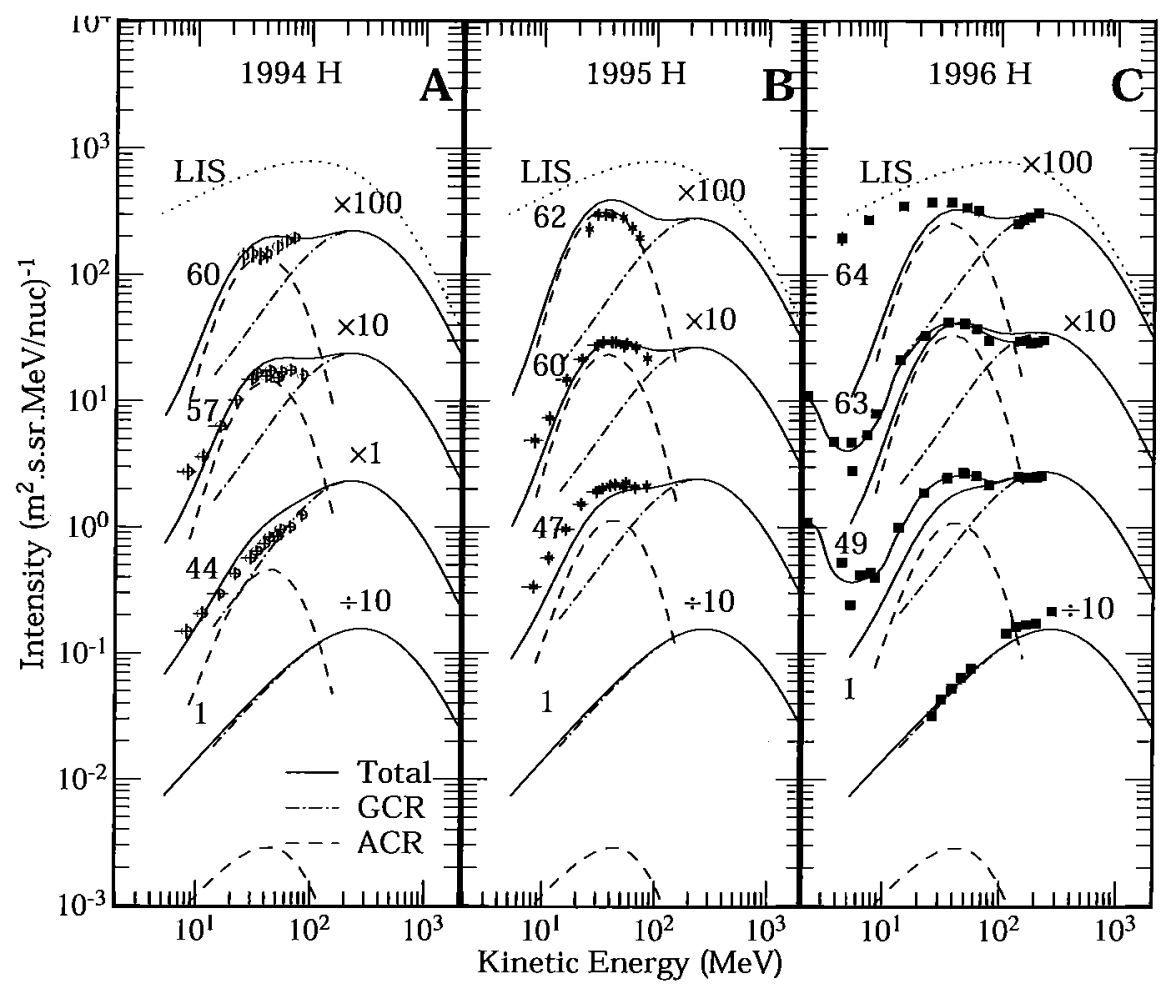

Figure 8 . Model fits to the 1994/5/6 $\mathrm{H}$ observations in the same format as the observations presented in Figure 3.3, showing the modulated ACR and GCR components at the spacecraft positions as indicated by the number to the left of each data set. The total modulated spectrum was calculated as the sum of the ACR and GCR components.

Table V

Kinetic energies (MeV/nuc) at which the ACR H contribution to the total $\mathrm{H}$ intensity is largest. The percentage contribution at this energy is shown in brackets.

\begin{tabular}{lllll}
\hline & IMP8 & V2 & V1 & P10 \\
\hline 1994 & $5.3(15 \%)$ & $23(59 \%)$ & $23(83 \%)$ & $23(85 \%)$ \\
1995 & $5.3(15 \%)$ & $23(69 \%)$ & $23(84 \%)$ & $23(88 \%)$ \\
1996 & $5.3(15 \%)$ & $23(73 \%)$ & $23(89 \%)$ & $23(88 \%)$ \\
\hline
\end{tabular}

demonstrates the "skin depth" effect for $10 \mathrm{MeV}$ particles: a large radial gradient for $r>60 \mathrm{AU}$, while the gradient for $r<60 \mathrm{AU}$ is largely, if not totally, governed by the adiabatic limit of modulation. In the adiabatic limit, the particle intensities are independent of radial distance. "Skin depth" effect is used somewhat analogous to a similar concept for radiation entering an object, where the skin depth into the object is the distance at which the intensity of the radiation reaches a value 


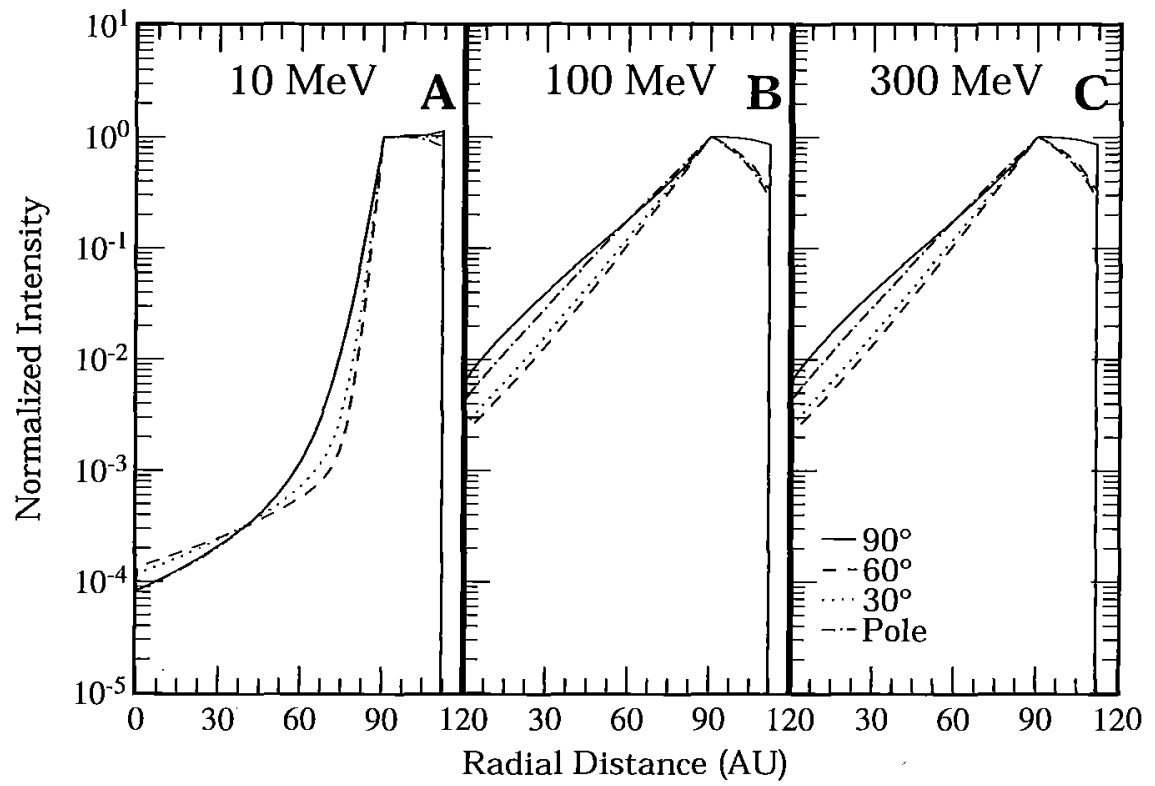

Figure 9. Radial intensity profiles for ACR H for four different polar angles, normalized at the SWTS intensity. Due to the same modulation parameters for all three periods, these profiles are identical for 1994, 1995, and $1996 \mathrm{ACR} \mathrm{H}$. These profiles are also identical to those calculated for ACR $\mathrm{He}^{+}$at $2.5,25$, and $75 \mathrm{MeV} /$ nuc and at 5,50 , and $150 \mathrm{MeV} /$ nuc for ACR $\mathrm{He}^{++}$.

of $1 / e$ of the intensity outside the object. The 100 and $300 \mathrm{MeV}$ radial profiles in panels B and $\mathrm{C}$ of this figure, respectively, are similar and show the intensity, $j$, increasing approximately proportional to radial distance, $r$, with the $300 \mathrm{MeV}$ intensities having a slightly larger gradient. The latitudinal dependence of the solar wind is largely responsible for the different profiles seen at higher latitudes. This is also illustrated by the latitudinal intensity profiles in Figure 11 which show a positive (small negative) latitudinal gradient in the outer (inner) heliosphere.

\subsubsection{Helium}

Following the method used in the previous paragraph for protons, we also calculated fits to the ACR and GCR He observations for the three observation periods. Using the same modulation parameters discussed in the previous paragraph, we found that the sum of a singly charged ACR component and the GCR component was unable to explain the observed ACR spectral peak width. We therefore included a doubly charged ACR $\mathrm{He}^{++}$component (as described by Jokipii (1996) and Mewaldt et al. (1996b)) in our calculations and used a larger value for $\kappa_{r r}$, as indicated in Table IV. We note that for $r \gg 1 \mathrm{AU}$ in a Parker spiral magnetic field, $\kappa_{\perp} \approx \kappa_{r r}=2.16 \times 10^{23} \beta P \mathrm{~cm}^{2} / \mathrm{s}$. For $1 \mathrm{GV}$ particles in the outer heliosphere, this is $68 \%$ of the value of $\kappa / \beta \approx 3 \times 10^{23} \mathrm{~cm}^{2} / \mathrm{s}$ used by Stone et al. (1996) to fit 1994 Voyager 1 and 2 ACR He and O observations. From this Table we also see 


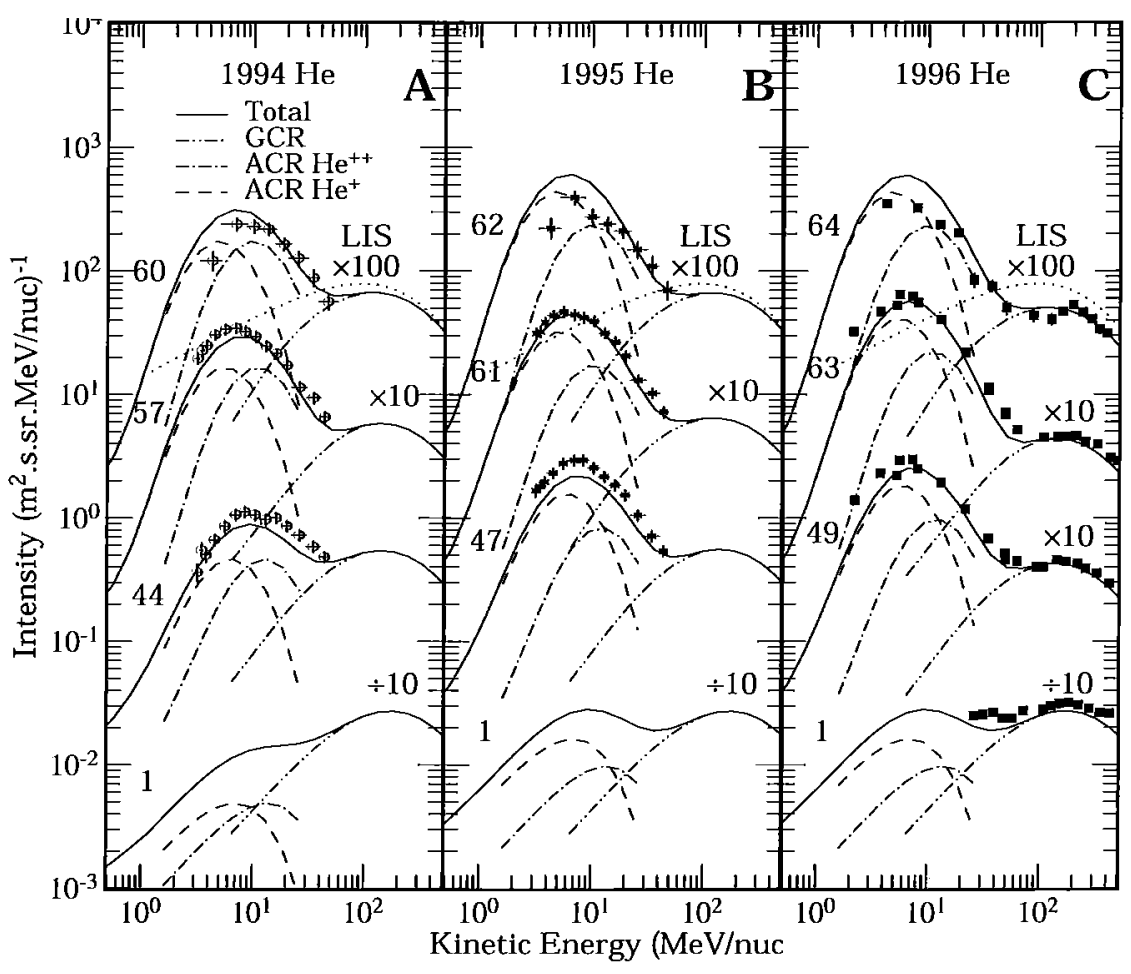

Figure 10. Model fits to the 1994/5/6 He observations in the same format as the observations presented in Figure 3.3, showing the modulated ACR $\mathrm{He}^{+}, \mathrm{He}^{++}$and GCR components at the spacecraft positions as indicated by the number to the left of each data set. The total modulated spectrum was calculated as the sum of the ACR and GCR components.

Table VI

Kinetic energies (MeV/nuc) at which the ACR He contribution to the total He intensity is largest. The percentage contribution at this energy is shown in brackets.

\begin{tabular}{lllll}
\hline & IMP8 & V2 & V1 & P10 \\
\hline 1994 & $0.57(46 \%)$ & $4.6(84 \%)$ & $3.2(92 \%)$ & $3.2(94 \%)$ \\
1995 & $0.57(46 \%)$ & $4.6(94 \%)$ & $3.2(97 \%)$ & $3.2(98 \%)$ \\
1996 & $0.57(53 \%)$ & $4.6(96 \%)$ & $3.2(98 \%)$ & $3.2(98 \%)$ \\
\hline
\end{tabular}

that the calculated source ratio of $\mathrm{He}^{+} / \mathrm{He}^{++}$increased from 1 in 1994 to 1.94 in 1995/1996.

Figure 10 shows that the fits to the observations are of similar quality to those for $\mathrm{H}$, but with the calculated ACR radial gradients somewhat larger than observed.

Table VI shows the energies at which the maximum ACR He contribution occurs for the calculated spectra. For $1 \mathrm{AU}$ these maxima of about $50 \%$ occur at $0.57 \mathrm{MeV} / \mathrm{nuc}$, while they occur at $\approx 4.6 \mathrm{MeV} / \mathrm{nuc}$ for Voyager 2 , and $3.2 \mathrm{MeV} / \mathrm{nuc}$ 


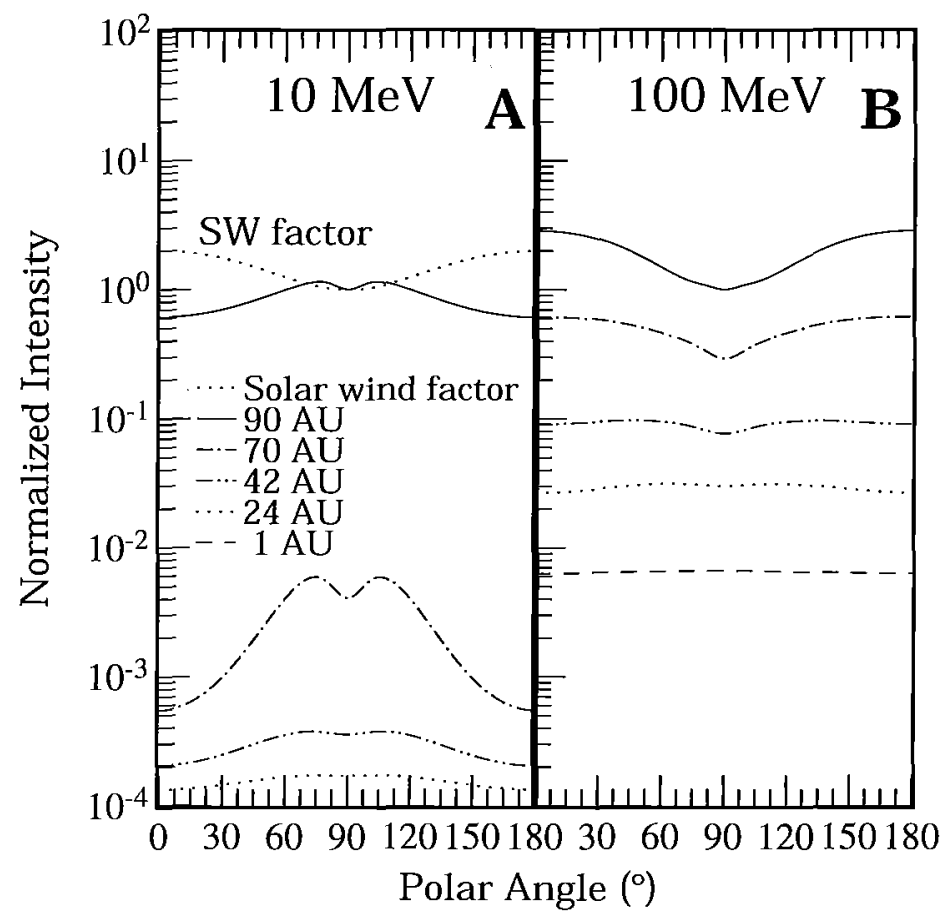

Figure 11. Latitudinal intensity profiles normalized to the intensity at the SWTS in the ecliptic plane. Due to the same modulation parameters for all three periods, these profiles are identical for 1994 , 1995, and $1996 \mathrm{ACR} \mathrm{H}$, and to those calculated for ACR $\mathrm{He}^{+}$at $2.5 \mathrm{MeV} / \mathrm{nuc}$ and $25 \mathrm{MeV} / \mathrm{nuc}$ or $5 \mathrm{MeV} / \mathrm{nuc}$ and $55 \mathrm{MeV} / \mathrm{nuc}$ for $\mathrm{ACR} \mathrm{He} \mathrm{H}^{++}$for these three periods. Also shown is the assumed latitude variation of the solar wind (SW factor)

for Voyager 1 and Pioneer 10. As with $\mathrm{H}$, the relative size of the ACR contribution increases towards the outer heliosphere, as well as from 1994 to 1996, with maximum calculated contributions of 98\% at Voyager 1 and Pioneer 10 in 1996.

\subsection{CONCLUSIONS}

We have shown that it is possible to obtain reasonable quality, simultaneous, fits to the outer heliospheric ACR and GCR H and He observations for 1994, 1995 and 1996 using a full-drift modulation model. To achieve these fits, (a) the diffusion coefficients were taken to be the same for the three periods, (b) the ACR source function was smaller in 1994 than in 1995/96, (c) the local interstellar spectrum was taken to be a third of generally acceptable values, and (d) the diffusion coefficients for He were larger than the corresponding values for $\mathrm{H}$. Point (c) is an indication that the diffusion coefficients used in these fits are too large for the GCR (higher rigidity) species. Point (d) is physically unacceptable because it implies different diffusion mean free paths for the two species. 
It was found that a doubly charged ACR $\mathrm{He}^{++}$component was required to explain the observed broad spectral peaks of ACR He. Furthermore, the ratio of ACR $\mathrm{He}^{+} / \mathrm{He}^{++}$was found to increase during the observation period (from 1994 to 1996). This result is, however, dependent on the rigidity dependence $(\beta P)$ of the diffusion coefficients: a flatter (steeper) rigidity dependence will broaden (steepen) the modulated ACR peak widths.

These conclusions indicate that the model is not yet self-consistent and that the magnitude and rigidity dependence of the diffusion coefficients have to be explored further.

\title{
4. The Acceleration of Pick-Up Ions at The Solar Wind Termination Shock: A Non-Linear Monte Carlo Calculation
}

\author{
F.C. Jones, M.G. Baring and D.C. Ellison
}

\subsection{INTRODUCTION}

We have employed our Monte Carlo simulation code (Ellison et al., 1996) to study the physical parameters that the solar wind termination shock would require to produce the observed anomalous cosmic ray hydrogen ions. As input data we have adopted the values of Vasyliunas and Siscoe (1976) for the interstellar ion flux in the heliosphere. These values were used by Cummings and Stone (1996), (see also Stone et $a l$., 1996), to determine the efficiency of acceleration of Anomalous Cosmic Rays by the solar wind termination shock. The work reported in this paper should be considered a preliminary study to explore the possibility that this shock can accelerate pick up ions to the observed ACR energies without the need for a preacceleration mechanism. We find that we can match the observed spectrum of hydrogen ions if we assume that the scattering is strong enough that $\lambda_{\|}=15 r_{g}$ where $\lambda_{\|}$is the scattering mean free path parallel to the mean magnetic field and $r_{g}$ is the particle's gyroradius. This level of turbulence is not excessive given that inferred in the vicinity of interplanetary shocks (Baring et al., 1997), and supernova shocks (Achterberg et al., 1994).

\subsection{THE PARAMETERS}

Following Cummings and Stone (1996) we take the shock position to be at 80 AU which leads to an obliquity (angle between the magnetic field and the shock normal) of $89^{\circ}$. The magnetic field is taken to be $0.05 \mathrm{nT}$, the solar wind hydrogen flux as $5 \times 10^{4} \mathrm{~cm}^{-2} \mathrm{~s}^{-1}$ and the flux of pick-up hydrogen to be $10240 \mathrm{~cm}^{-2} \mathrm{~s}^{-1}$. The solar wind speed is taken to be $500 \mathrm{~km} / \mathrm{s}$ and the pickup ions are assumed to have a "flat top" distribution extending from almost zero velocity to twice the solar wind speed. This gives for the sonic and Alfvénic Mach numbers $M_{S}=6.85$ and 


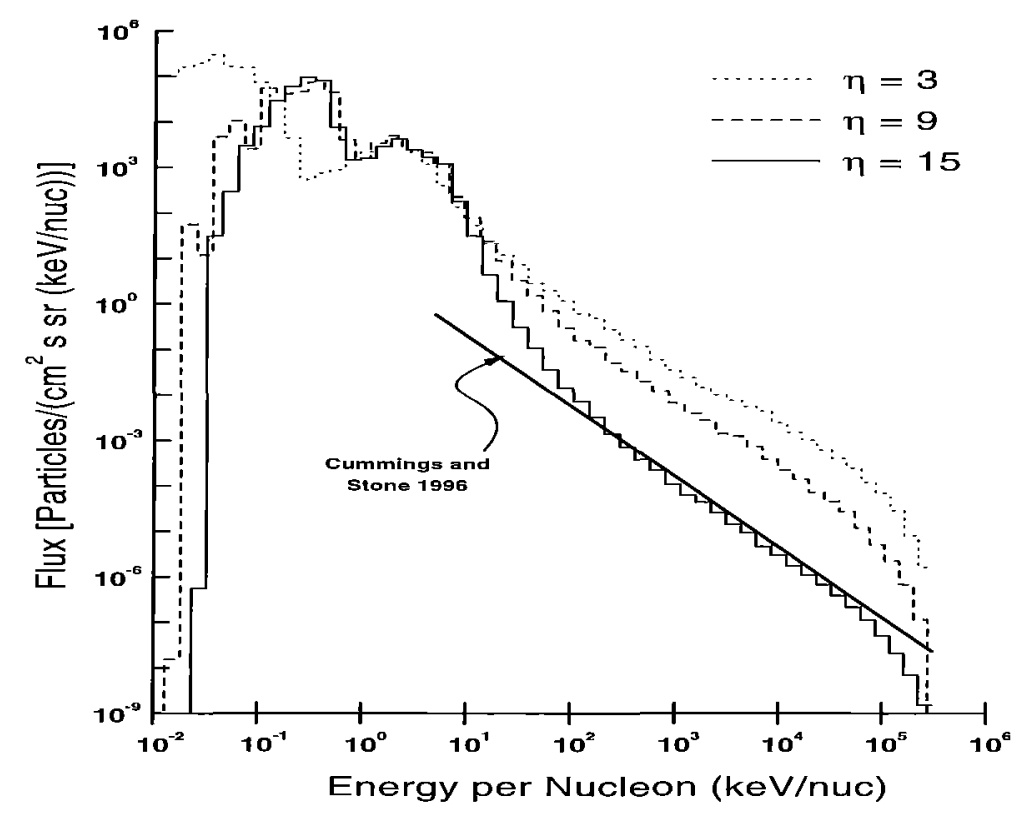

Figure 12. Anomalous hydrogen flux for different scattering rates. The solid line is the prediction (Cummings and Stone, 1996) of the spectrum at the shock from a fit to the data.

$M_{\text {Alf }}=15.9$. We chose values of the phenomenological parameter $\eta=\lambda_{\|} / r_{g}$ of 3,9 , and 15 and found the best fit for $\eta=15$.

\subsection{RESULTS}

The simulation was run self-consistently for hydrogen which is the major component and then helium and oxygen were accelerated in this shock in the test particle mode. Helium is about one sixth of the hydrogen content in the pick-up ions but as it has four times the mass it should be included in the shock structure calculation. Such calculations, however, take a considerable amount of time so for this preliminary study they were not included at this time.

In Figure 12 we see the spectra produced by the various values of $\eta$. It is clear that $\eta=15$ fits the predicted shock spectrum best. Such a low value of scattering does not produce very much modification of the shock as can be seen from Figures 13 and 14.

Since the particles' mean free path scales as $\lambda \propto P$, the diffusion scales for oxygen and helium are such that at $10 \mathrm{keV}$ per nucleon, which is the energy of the pick up ions' quasi-thermal peak behind the shock, these nuclei see all or 


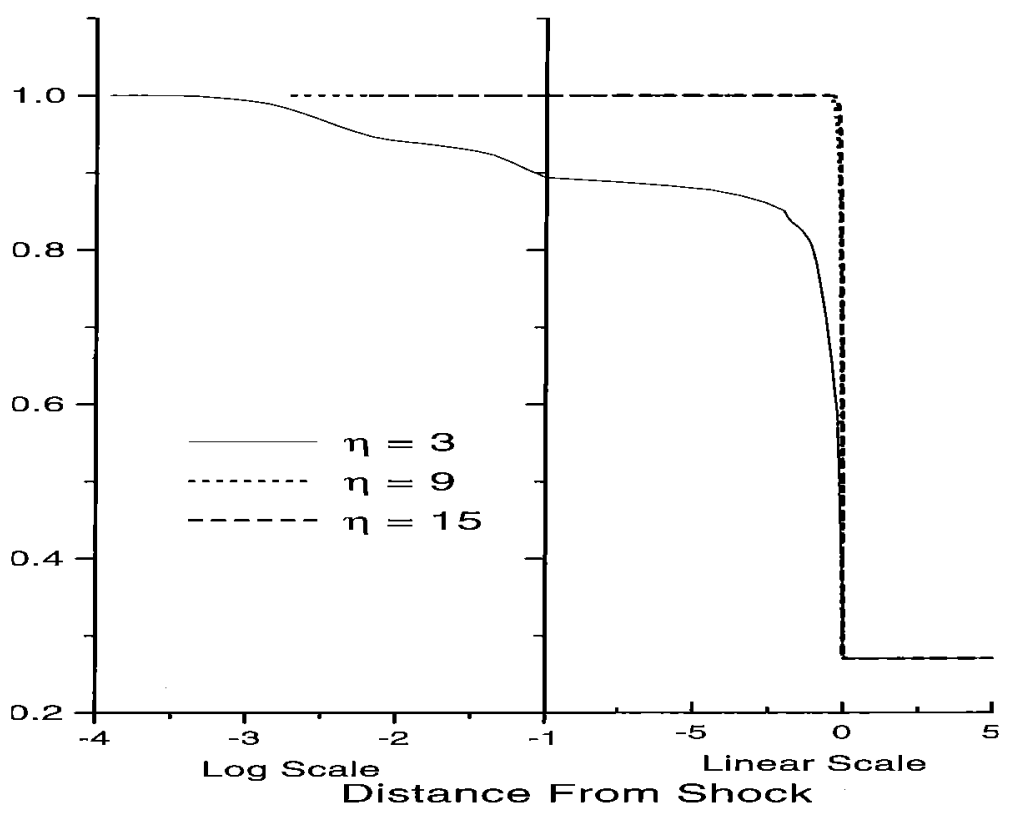

Figure 13. Shock velocity profiles for different scattering rates.

most of the shock as a sharp transition whereas the hydrogen sees an extended structure. This produces an enhancement in these elements (Ellison et al., 1981) over hydrogen as can be seen from Figure 15.

The oxygen should be enhanced more than the helium by the above reasoning. At the present time we do not understand why the opposite appears to happen. A detailed examination of the spectra suggests that a statistical fluctuation at lower energies may be the culprit. Such low energy fluctuations in the low energy part of a spectrum can cause large variations at the high energy end. More time consuming runs with better statistical accuracy will be run in the future to examine this possibility. In the meantime the present calculations show that the termination shock is quite capable of producing the observed spectra of anomalous cosmic ray ions with reasonable values of the shock parameters. 


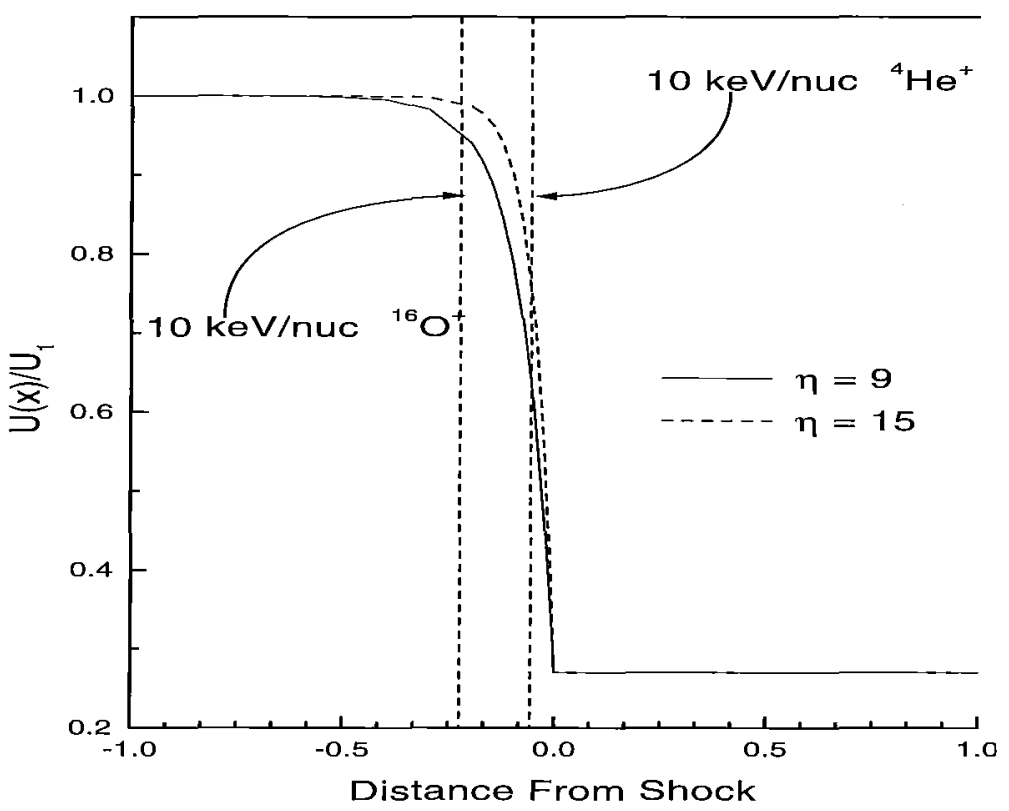

Figure 14. Expanded shock velocity profiles for two scattering rates. The dotted vertical lines represent the upstream diffusive length scales for singly charged, $10 \mathrm{keV}$ per nucleon helium and oxygen. The diffusive length for hydrogen is one fourth that of the helium.

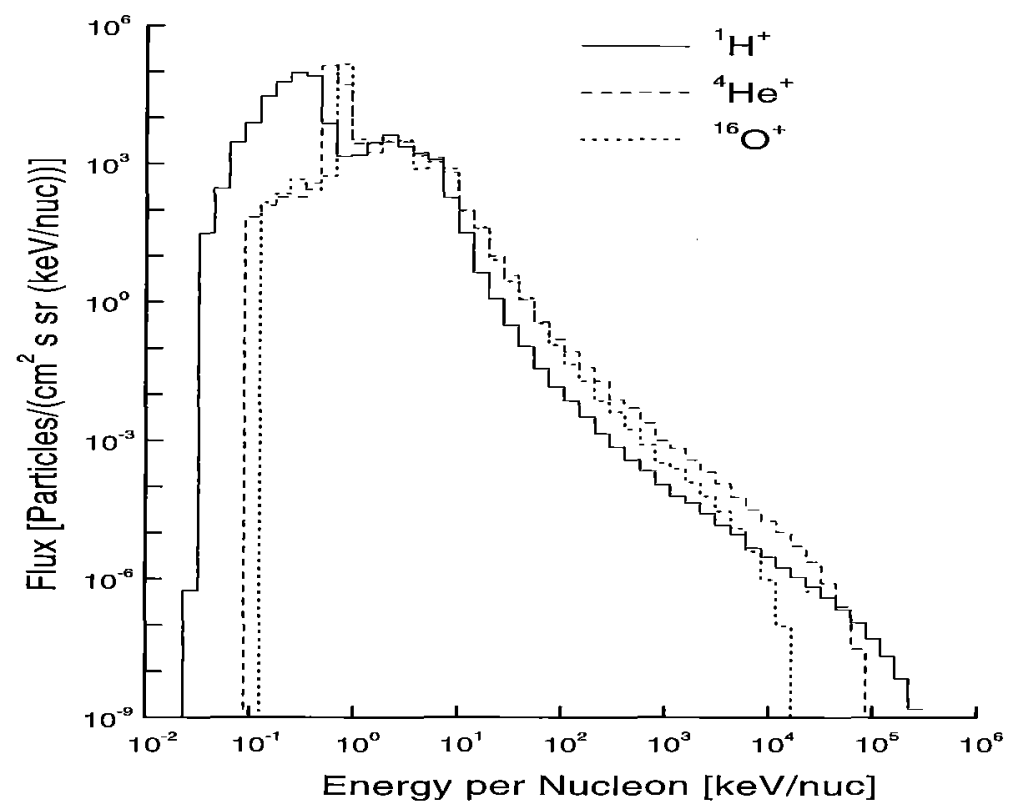

Figure 15. Normalized spectra of singly charged hydrogen, helium, and oxygen for a scattering rate of $\eta=15$. The different elements were all normalized to have the same total flux to show the enhancement of ${ }^{4} \mathrm{He}^{+}$and ${ }^{16} \mathrm{O}^{+}$over hydrogen. 


\title{
5. Injection and Acceleration of Pickup Ions at the Termination Shock
}

\author{
J. Giacalone and J.R. Jokipii
}

\subsection{INTRODUCTION}

The association between anomalous cosmic rays and interstellar pickup ions is well established (Fisk et al., 1974). Moreover, the source of ACR is beyond current spacecraft and is certainly the termination shock of the solar wind (Pesses et al., 1981). However, the energy of freshly ionized pickup ions is some 5 orders of magnitude smaller than ACR energies, which raises the question of how the acceleration occurs. It has been debated for some time whether low-energy pickup ions are accelerated with great enough efficiency to account for the observed intensity of anomalous cosmic rays. Some initial acceleration, via some process in the inner heliosphere for example, may be required.

Here we address the physics of the injection of pickup ions locally at the termination shock using a numerical simulation model which treats the pickup ions as test particles interacting with actual magnetic turbulence. This approach treats the scattering of particles more accurately than models using phenomenological scattering. Because the nature of the turbulence at the termination shock is not known, different turbulence spectra are used.

\subsection{Perpendicular TRANSPORT IN SHOCK ACCELERATION}

Charged particles that interact with collisionless shocks may be accelerated to speeds that are much larger than the speed of the shock. If the energy density contained in the random magnetic turbulence is of the same order, or smaller than, the energy density contained in the mean magnetic field, the direction of the mean field with respect to the unit normal to the shock is an important parameter which determines the efficiency at which the incident charged particles will be accelerated. For quasi-parallel shocks, the mean field points nearly to the same direction as the shock normal while for quasi-perpendicular shocks the opposite is true. Because particles freely move along the magnetic field lines and scatter (or "mirror") occasionally back and forth, quasi-parallel shocks are generally more capable of accelerating initially low-energy particles. On the other hand, because magnetic field lines are frozen into the plasma, it is difficult to accelerate lowenergy particles at quasi-perpendicular shocks because the particles do not move normal to the field rapidly enough to stay near the shock. This is the well-known injection problem.

The fundamental issue in the injection problem is whether there is enough diffusion normal to the shock to keep the particles near the shock. If sufficient diffusion exists, one may use the transport equation to describe the spatial transport and acceleration of the particles (Parker, 1965). Jokipii (1991) derived a parameter 

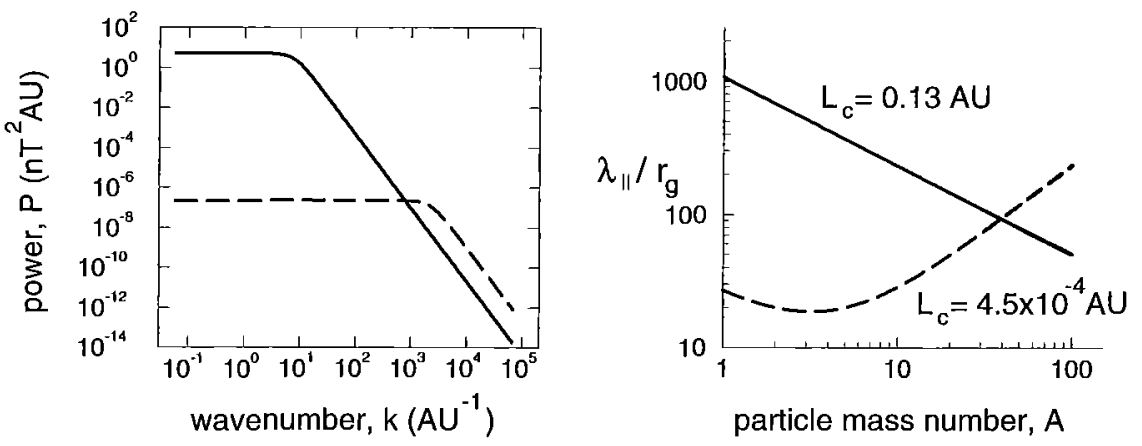

Figure 16. Left: Synthesized IMF power spectrum at $80 \mathrm{AU}$ used in the numerical simulations. Right: ratio of the parallel mean-free path to particle gyroradius versus particle mass based on the quasi-linear theory using the power spectra shown to the left.

which describes the ability for particles to remain near the shock in terms of the diffusion coefficient normal to the shock, $\kappa_{x x}$.

$$
=\frac{U r_{g}}{\kappa_{x x}} \ll 1 \quad \Rightarrow \quad \text { large diffusion }
$$

where $U$ is the upstream plasma speed relative to the shock, and $r_{g}$ is the Larmor radius. Physically, (4) represents the ratio of the flow speed to the maximum "diffusive" speed of the particles.

The physics of the transport of charged-particles across a magnetic field is currently not well understood. Two types of cross-field diffusion have been recognized: (a) so-called "billiard-ball" scattering in which a particle scatters across the field one gyroradius for every mean-free path measured along the field $\lambda_{\|}$, and (b) the case where the particle motion is mostly influenced by the random motion of magnetic-field lines. For case (a) the form of the cross-field diffusion coefficient is well known; however, for case (b), which probably dominates the perpendicular transport of low-energy particles, there are a number of theories but no consensus. We assume here that for case (b) the perpendicular transport is some fraction $\eta(<1)$ of the parallel transport. Consequently, for the two types of cross-field diffusion, (4) becomes

$$
= \begin{cases}3(U / v)\left(\lambda_{\|} / r_{g}\right) & \text { billiard-ball scattering } \\ (3 / \eta)(U / v)\left(r_{g} / \lambda_{\|}\right) & \text {field-line mixing }\end{cases}
$$

where $v$ is the particle speed, $\lambda_{\|}$is the parallel mean-free path, and $\eta$ is the ratio of perpendicular to parallel transport coefficients.

The right panel of Figure 16 is the ratio of mean-free path to particle gyroradius as a function of particle mass ( $A$ is the ratio of particle mass to proton mass), determined from the quasi-linear theory (e.g. Earl, 1974) using the two power spectra shown in the left panel. 
The perpendicular transport of particles whose inverse gyroradius is at a point in the power spectrum that is falling off rapidly with wavenumber $k$ will be influenced mostly by the field-line mixing; while for those whose resonant wavenumber is in the flat portion of the power spectrum will cross-field diffuse mainly via billiardball-type scattering.

The efficiency at which pickup ions are accelerated by the nearly perpendicular termination shock will depend on. The smaller this value, the more likely that the distribution function can be described by the transport equation, and, therefore, the particles are readily accelerated to high energies as in the standard diffusive shock acceleration theory (e.g. Jokipii, 1987). Judging from Equation (5) and the right panel of Figure 16, increases with $A$ (note that small $A$ particles have smaller gyroradii and are in the field-line mixing regime). Consequently, the simple view that pickup ions are accelerated via scattering off of convecting magnetic irregularities at a planar collisionless shock leads to the conclusion that the acceleration of lighter particles are more efficient than heavier particles. This result is contrary to the interpretation of Voyager ACR observations by Cummings and Stone (1996).

\subsection{NUMERICAL RESULTS}

Shown in Figure 17 are the results of three separate numerical simulations of the interaction of freshly-ionized pickup ions (helium and oxygen) with the termination shock. The details of the shock model are described in Giacalone and Jokipii (1996). The incident pickup-ion distributions were obtained by using the model of Vasyliunas and Siscoe (1976) for a termination shock at $80 \mathrm{AU}$ along the direction of the flow of the neutrals into the heliosphere. The incident fluxes of the two species are the same as those quoted by Cummings and Stone (1996). The circlesymbols on these plots are portions of the data presented by Cummings and Stone (1996) for anomalous cosmic rays. These were chosen because they are no longer increasing with radial distance. The upper three panels are for $\mathrm{He}^{+}$ions, while the lower three panels are $\mathrm{O}^{+}$. Each set of three panels are for different parameters for the turbulence as indicated at the top.

Not much is known about the IMF turbulence spectra in the outer heliosphere. From Ulysses observations in the inner heliosphere (e.g. Smith et al., 1995; Jokipii et al., 1995) we know that long-wavelength fluctuations have a variance that falls off as $r^{-2}$, whereas short wavelengths fall off as $r^{-3}$. Assuming then that the flat part of the power spectrum (see Figure 16) falls off as $r^{-2}$ and the high- $k$ portion falls off as $r^{-3}$, and using typical IMF parameters at $1 \mathrm{AU}$, we can extrapolate the spectrum to $80 \mathrm{AU}$. We find that the correlation length, $L_{c}$, is of the order of 0.1 AU. Shown in the far left and far right panels are numerical results using a correlation length of $L_{c}=0.13 \mathrm{AU}$ with two different variances; while for the middle panel a correlation length equal to the gyroradius of pickup protons, which may characterize turbulence self-generated by the dominant pickup protons, is used. 


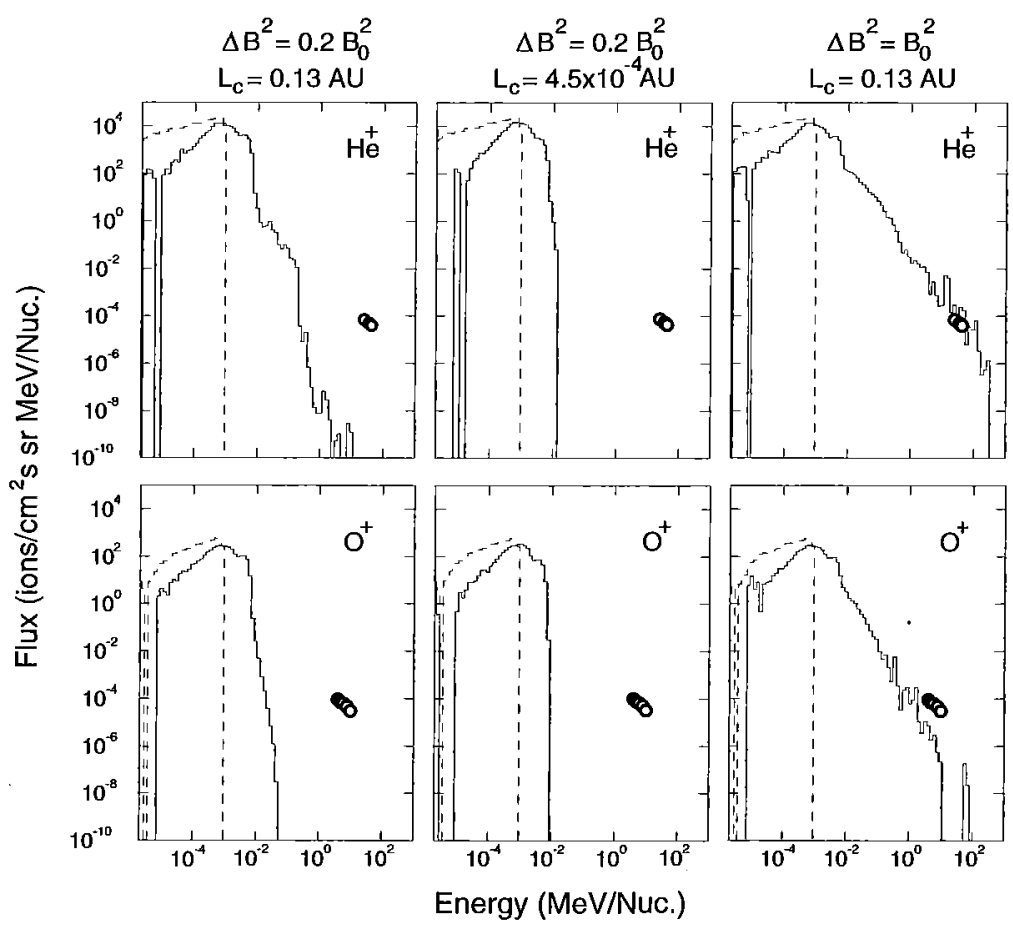

Figure 17. Simulated energy spectra of helium and oxygen at the termination shock. The dashed lines are the input spectra and the circle symbols are Voyager observations of anomalous helium and oxygen.

Figure 17 shows that only for the case of strong magnetic turbulence having a long correlation length does pickup helium get accelerated efficiently enough to account for the observed fluxes of anomalous $\mathrm{He}^{+}$. Although oxygen is accelerated more efficiently compared to the other turbulence spectra, it is less efficiently accelerated than $\mathrm{He}^{+}$(note that the simulated He intensity matches the observations while $\mathrm{O}$ does not). In the other two forms of the turbulence spectrum, neither species is accelerated to anomalous cosmic-ray energies.

\subsection{DISCUSSION AND SUMMARY}

We have considered the physics of the acceleration of pickup ions by the termination shock from the viewpoint that the particles interact with magnetic irregularities embedded in the solar wind. Under some conditions the pickup ions may be accelerated some 4-5 orders of magnitude to anomalous cosmic-ray energies. However, strong levels of long-wavelength turbulence are needed to account for the observed intensities of anomalous cosmic rays. Additionally, lighter particles are more efficiently accelerated than heavier particles which is contrary to the interpretation of Voyager observations reported by Cummings and Stone (1996). 


\title{
6. The Self-Consistent Stochastic Pre-Acceleration of Pickup Ions in the Heliosphere
}

\author{
J.A. le Roux and V.S. Ptuskin
}

\subsection{INTRODUCTION}

The purpose of this work is to reinvestigate the self-consistent stochastic preacceleration (2nd order Fermi) of pickup ions in the solar wind upstream of the heliospheric termination shock. The term "self-consistent" refers here to the determination of the spectrum of low frequency magnetohydrodynamic (MHD) waves with which the pickup ions interact resonantly. The model proposed here attempts to address some of the shortcomings in previous self-consistent work (see Bogdan et al., 1991): (i) It includes the effect of adiabatic cooling on pickup ions. This mechanism competes against stochastic acceleration and will allow us to determine the pre-accelerated spectrum more realistically. (ii) It simulates the effect of wavewave interactions as a non-linear diffusion process in wave number space ( $k$-space) following the example of Zhou and Matthaeus (1990). Wave damping competes against wave cascading and will allow us to investigate the consequent suppression of the stochastic pre-acceleration of pickup ion $\mathrm{H}^{+}$compared with heavier elements. The aim is to see whether the cause for the observed under-abundance of anomalous cosmic ray (ACR) $\mathrm{H}^{+}$(Cummings and Stone, 1996) has its origin upstream of the heliospheric termination shock. For a discussion of this underabundance occurring at the heliospheric termination shock, see Section 4. (iii) Our model makes a distinction between accelerated spectra in the quiet solar wind, and inside co-rotating merged interaction regions (CMIRs) where the heliospheric magnetic field (HMF) is usually stronger.

\subsection{THE MODEL}

The model is used to study the self-consistent transport of $\mathrm{H}^{+}, \mathrm{He}^{+}$and $\mathrm{O}^{+}$pickup ions in the upwind direction along the equatorial plane upstream of the heliospheric termination shock. The transport equation for an isotropic distribution of pickup ions $f(r, p, t)$ in the solar wind at radial distance $r$, with momentum $p$ and time $t$ is given by the following equation in spherical symmetry (see e.g., Isenberg, 1987):

$$
\frac{\partial f}{\partial t}+u \frac{\partial f}{\partial r}-\frac{1}{p^{2}} \frac{\partial}{\partial p}\left(p^{2} D_{p p} \frac{\partial f}{\partial p}\right)-\frac{1}{3 r^{2}} \frac{\partial}{\partial r}\left(r^{2} u\right) p \frac{\partial f}{\partial p}=Q_{\text {pui }} \text {. }
$$

Here, $u$ is the solar wind flow speed, and $D_{p p}$ is the momentum diffusion coefficient for stochastic acceleration given by

$$
D_{p p}=2 \pi^{2} q^{2}\left(\frac{v_{A}}{c}\right)^{2} \frac{1}{v} \int_{k_{\circ}}^{\infty}\left(1-\left[\frac{k_{\circ}}{k}\right]^{2}\right) \frac{W_{k}}{k} d k
$$

where $q$ is the net charge of the pickup ions, $v_{A}$ is the Alfvén speed, $c$ is the speed of light, and $k_{\mathrm{o}}=/ v(>0$ is the particle's gyrofrequency and $v$ its speed) is 
the minimum cyclotron resonant wave number for wave-particle interactions. $W_{k}$ is the spectral energy density of MHD waves with which pickup ions resonate at wave number $k=/(v|\mu|)$ where $\mu$ is the cosine of the pickup ion pitch angle. This equation, which follows from standard quasi-linear theory (e.g., Schlickeiser, 1989), describes the pickup ion momentum diffusion due to its interaction with circularly polarized, dispersionless MHD waves propagating along the HMF at the Alfvén speed with equal intensities in the forward and backward directions. The source term $Q_{\text {pui }}$ represents a spherical shell of freshly ionized pickup ions in velocity space continuously injected throughout the heliosphere at a speed $v=u$. The calculation of the source term is based on standard values for the interstellar neutral density which are $0.077,0.013,6.2 \times 10^{-4}$ particles $\mathrm{cm}^{-3}$, and ionization frequencies at Earth of $5 \times 10^{-7}, 6.8 \times 10^{-8}, 5 \times 10^{-7} \mathrm{~s}^{-1}$ for $\mathrm{H}^{+}, \mathrm{He}^{+}$and $\mathrm{O}^{+}$ pickup ions, respectively.

The MHD wave spectrum is described by a transport equation for spectral wave energy density $W_{k}(k, r, t)$ (see e.g., Miller and Roberts, 1995) given by

$$
\frac{\partial W_{k}}{\partial t}-\frac{\partial}{\partial k}\left(D_{k k} \frac{\partial W_{k}}{\partial k}\right)-\gamma_{k} W_{k}=Q_{k} .
$$

In this equation, the second term on the left approximates the complex process of wave-wave interactions as a non-linear diffusion process in $k$-space where $D_{k k}\left(W_{k}, k, t\right)$ denotes the non-linear diffusion coefficient. This diffusion coefficient can be written as follows for the case of Alfvénic turbulence:

$$
D_{k k}=\frac{C_{k}}{2 \pi} \frac{v_{A}}{\sqrt{2 \bar{P}_{B}}} k^{7 / 2} W_{k}^{1 / 2},
$$

where $C_{k}$ is the Kolmogorov constant of the order of 1 , and $P_{B}=B^{2} /(8 \pi)$ the pressure in the large-scale HMF. The last term on the left describes wave damping and is given by

$$
\gamma_{k}=-2 \pi^{2} q^{2}\left(\frac{v_{A}}{c}\right)^{2} \frac{1}{k} \sum_{s} \int_{p_{\circ}}^{\infty} 4 \pi p f_{s} d p
$$

where $f_{s}$ is the isotropic distribution function for different pickup ion species $s$ as found from the solution of equation (6), and $p_{\circ}=m / k$ is the minimum resonant momentum $(\mu=1)$ with $m$ the mass of the pickup ions. To solve the wave equation, an undamped Kolmogorov spectrum $\left(W_{k} \propto k^{-5 / 3}\right)$ is specified as an initial condition instead of specifying $Q_{k}$. It is assumed that wave-particle interactions are associated with slab turbulence so that an appropriate value for the amplitude of the transverse field fluctuations is $\left\langle\left(\delta B_{\perp}\right)^{2}\right\rangle / B^{2}=0.2$. For wave-wave interactions the two-dimensional (2D) component is also included and $\left\langle\left(\delta B_{\perp}\right)^{2}\right\rangle / B^{2}=1.49$ (Bieber et al., 1995) which signifies strong Kolmogorov turbulence. 

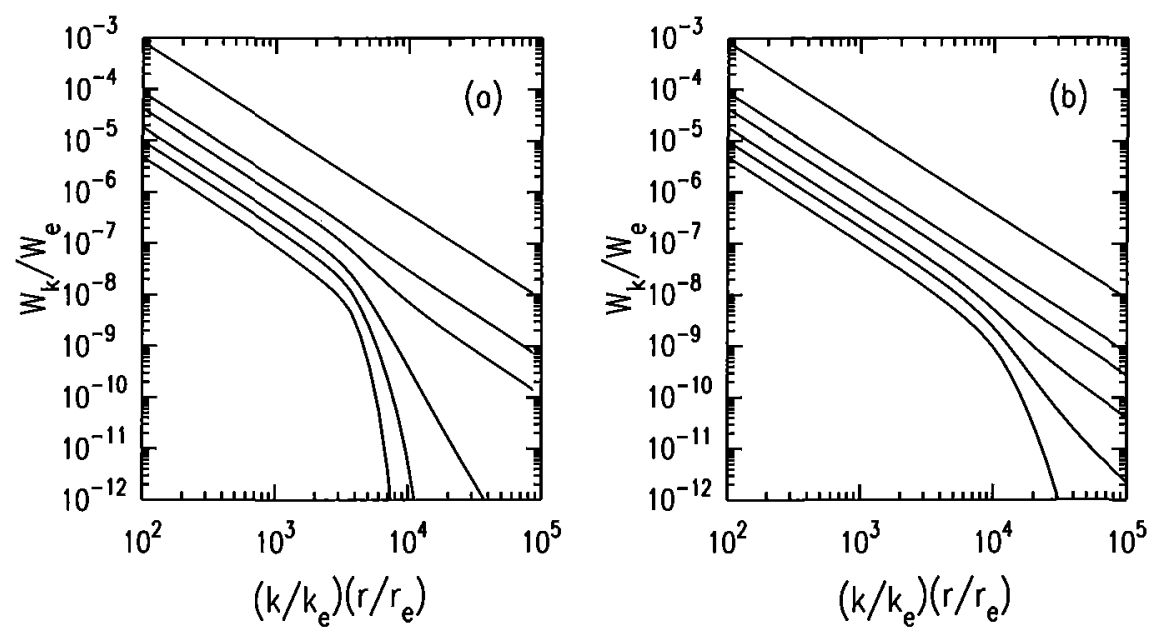

Figure 18. (a). The calculated spectral energy density $W_{k}$ as a function of wave number $k$. Here, $W_{k}$ is normalized to its assumed value $W_{e}\left(W_{e}=2.5 \mathrm{erg} \mathrm{cm}^{-2}\right)$ at the principal wave number $k_{e}$ $\left(k_{e}=2.2 \times 10^{-12} \mathrm{~cm}^{-1}\right)$ at Earth, and $k$ to the principal wave number at Earth. The curves from top to bottom denote $2,5,10,23,42$ and $85 \mathrm{AU}$ along the equatorial plane, respectively. Each curve's $k$-values are multiplied with $r$ normalized to the Sun-Earth distance. These results are valid in quiet solar wind regions $\left(B / B_{p}=1\right)$. (b). As (a), but denote wave spectra inside CMIRs $\left(B / B_{p}=3\right)$.

\subsection{RESULTS AND DISCUSSION}

In Figure 18 we show self-consistently calculated MHD wave spectra at different radial distances in the upwind direction along the equatorial plane. Figure 18(a) is valid in the quiet solar wind where it is assumed that $B / B_{p}=1$ ( $B_{p}$ is the Parker HMF magnitude). Figure 18(b) is calculated for a compressed $\operatorname{HMF}\left(B / B_{p}=3\right)$ which is an appropriate value for inside CMIRs (Burlaga et al., 1997).

This implies a factor of 9 increase in the spectral wave energy density. It is assumed that $C_{k}=3$. This value is motivated as follows: (i) The numerical MHD turbulence modelling by Verma et al. (1996) shows this constant to be larger than one for 2D turbulence as assumed for wave cascading in this model. (ii) For $C_{k}=1$ wave damping shows up strongly inside $5 \mathrm{AU}$ from the Sun contrary to observations which only mention possible pickup ion wave generation. For more information, read the figure captions.

The main result in Figure 18(a) is that strong wave damping due to the pickup ion $\mathrm{H}^{+}$occurs beyond $10 \mathrm{AU}$ from the Sun for normalized $k r>4 \times 10^{3}$ where wave damping dominates wave cascading. The minimum normalized $k$-value for wave-particle resonance is $k=5.4 \times 10^{3}$ in the case of unaccelerated $\mathrm{H}^{+}$pickup ions. The occurrence of significant wave damping for $k r<5.4 \times 10^{3}$ is caused by the stochastic acceleration of pickup ion $\mathrm{H}^{+}$which lowers the minimum $k$ value for wave-particle resonance. In the paper by Bogdan et al. (1991), strong wave damping is already apparent for $r<10 \mathrm{AU}$. This illustrates the difference 

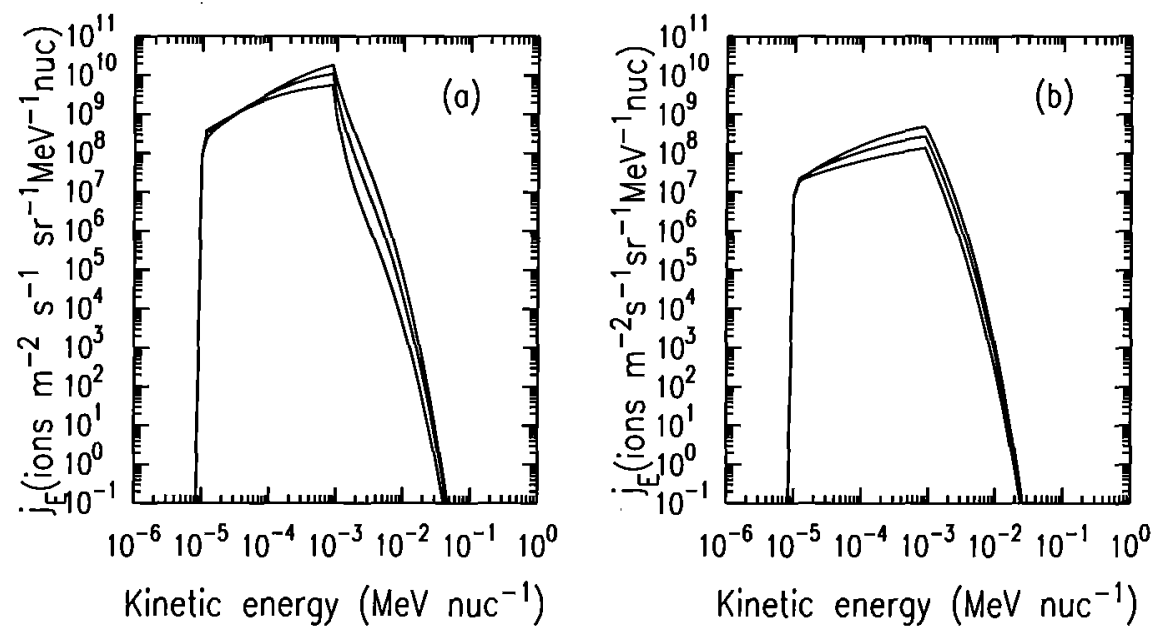

Figure 19. (a). Simulated pickup ion $\mathrm{H}^{+}$differential intensities (ions $\mathrm{m}^{-2} \mathrm{~s}^{-1} \mathrm{sr}^{-1}(\mathrm{MeV} / \mathrm{nuc})^{-1}$ in the quiet solar wind as a function of kinetic energy ( $\mathrm{MeV} / \mathrm{nuc}$ ). From top to bottom the curves represent $23,42,85 \mathrm{AU}$, respectively. (b). As (a), but for pickup ion $\mathrm{He}^{+}$.

when wave-wave interactions are allowed to compete against wave damping. Small deviations from the Kolmogorov spectral slope are also detectable in the curves beyond $10 \mathrm{AU}$ for $k r>1 \times 10^{3}$ due to wave damping by the pickup ion $\mathrm{He}^{+}$, while the damping effects from the pickup ion $\mathrm{O}^{+}$are negligible. This follows because: (i) These pickup ions are less numerous than the pickup ion $\mathrm{H}^{+}$so that $\gamma_{k}$ is smaller. (ii) They resonate with MHD waves at lower $k$-values where the process of wave cascading is much stronger. The Bogdan et al. (1991) model exhibits clear wave damping effects by helium pickup ions at $1 \mathrm{AU}$, but without the contribution of strong wave-wave interactions. Figure 18(b), associated with CMIRs, shows strong damping effects only for $r>23 \mathrm{AU}$, and for $k$-values larger by a factor of 3 , which reflects the increase in the minimum $k$-value for wave-particle resonance in response to the larger HMF magnitude inside CMIRs.

In Figure 19 we display calculated pickup ion spectra in the quiet solar wind for $\mathrm{H}^{+}$(Figure 19a) and $\mathrm{He}^{+}$(Figure 19b). For more details, see the figure captions. For pickup ions with kinetic energy $\left(E_{k}\right)>1 \mathrm{keV} /$ nuc, the $\mathrm{He}^{+}$and $\mathrm{O}^{+}$spectra (not shown) have the typical exponential spectra as expected in the absence of wave damping (e.g., Isenberg, 1987). This is not surprising, given the inability of these species to damp the wave spectra. The pickup ion $\mathrm{H}^{+}$spectra in Figure 19(a) differ markedly from the spectra of the two other species. For $1 \mathrm{keV}<E_{k}<5 \mathrm{keV}$, the spectra become increasingly concave with increasing $r$. For $E_{k}>5 \mathrm{keV}$ the spectra resume the typical exponential behavior. This concavity in the spectra is a direct consequence of the strong wave damping caused by the pickup ion $\mathrm{H}^{+}$ which reduces the effectiveness of the acceleration of pickup ion $\mathrm{H}^{+}$. The number of pickup ions with $E_{k}>5 \mathrm{keV}$ are not sufficient to damp the wave spectra 

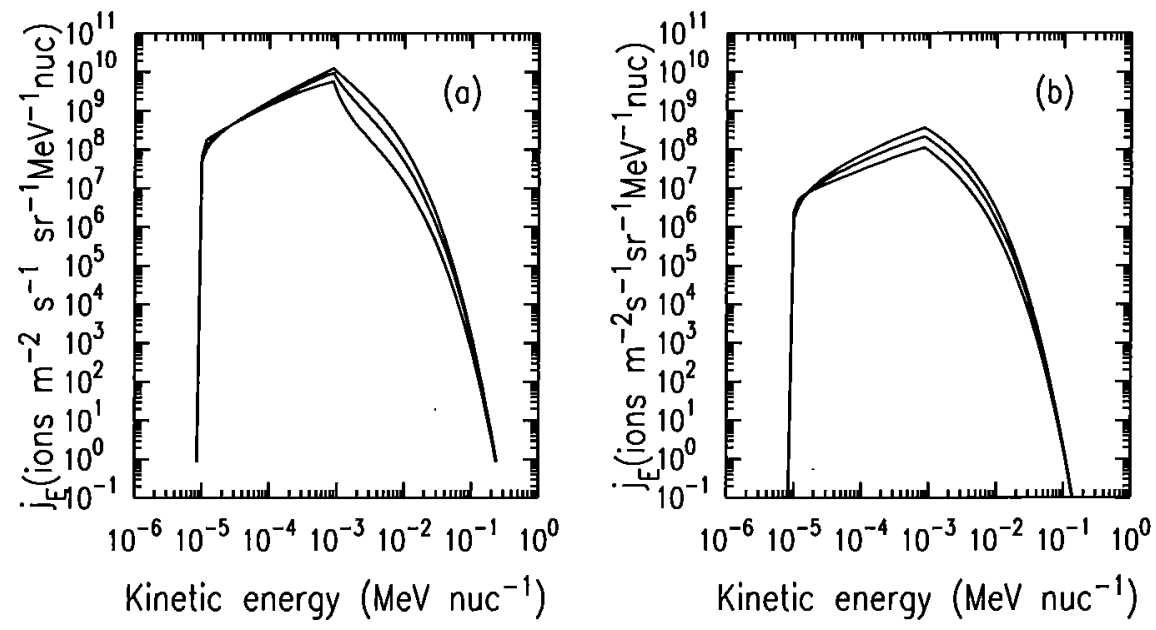

Figure 20. This figure has the same format as Figure 19, but calculations are valid inside CMIRs.

significantly, and they get accelerated by that part of the wave spectrum which still reflects well developed Kolmogorov turbulence. Overall, the acceleration is rather weak in the quiet solar wind with the pickup ions reaching a speed of $v \approx 3 u$. In Figure 20 we show pickup ion spectra as we expect them to be inside CMIRs at large $r$. In this case, the pickup ions are accelerated more efficiently, acquiring speeds of $v \approx 8 u$. The $\mathrm{H}^{+}$spectra become concave farther away from the Sun at $r>20 \mathrm{AU}$. The acceleration efficiency is clearly less affected inside CMIRs because the damping occurs at larger $k$-values and larger $r$ (see equation 8).

\subsection{INTERPRETATION}

For pickup ions to be injected into the process of diffusive shock acceleration at the quasi-perpendicular heliospheric termination shock and become ACRs, it is needed that they should have speeds $v>u_{1}$ where $u_{1}$ is the upstream solar wind convection speed. This requirement is based on the assumption that effective cross-field scattering of pickup ions by magnetic irregularities takes place near the heliospheric termination shock. Only then will they be able to move back upstream to interact with upstream waves and have multiple shock encounters. Therefore, we assume simply for illustration that the seed population of ACRs consists of pre-accelerated pickup ions with $v>x u_{1}$ where $x$ is an unknown parameter. We calculate the density in the seed populations of pickup ion $\mathrm{H}^{+}$and $\mathrm{He}^{+}$for different values of $x$ to see how the density ratio varies relative to the density ratio of the full pickup ion distributions. Using standard parameters, Cummings and Stone (1996) found the density ratio of $\mathrm{H}^{+} / \mathrm{He}^{+}$in pickup ions to be $\approx 45$. They observed this ratio as $\approx 6$ for ACRs, implying an under abundance of a factor of $\approx 7.5$ in ACR $\mathrm{H}^{+}$. We find that if the seed populations come from that part of the pre-accelerated pickup ion spectra where pickup ions have speeds $v>1.5 u_{1}$ at $85 \mathrm{AU}$ in the 
quiet solar wind, then the ratio of $\mathrm{H}^{+} / \mathrm{He}^{+}$is as low as $\approx 2.6$, which means an under-abundance by a factor of $\approx 17$ in $\mathrm{ACR} \mathrm{H}^{+}$. In the quiet solar wind the pre-acceleration of pickup ion $\mathrm{H}^{+}$can be more than sufficiently suppressed due to pickup ion $\mathrm{H}^{+}$wave damping to explain the observed under-abundance in ACR $\mathrm{H}^{+}$. Inside CMIRs the ratio of the seed population is $\approx 13$ for $v>1.5 u_{1}$, a factor of $\approx 2$ higher than the observed ratio for ACRs. It may well be that, averaged over both CMIR and quiet solar wind regions, the observed under abundance will be found, given that the seed population starts at $v>1.5 u_{1}$.

\title{
7. The Pre-Acceleration of Anomalous Cosmic Rays in the Inner Heliosphere
}

\author{
J. Giacalone and J.R. Jokipii
}

\subsection{INTRODUCTION}

It is known that the intensity of particles with energies lower than that of anomalous cosmic rays are mainly produced in the inner heliosphere. They are composed of solar energetic particles, accelerated interstellar pickup ions, and accelerated pickup ions from other sources (c.f. Geiss et al., 1995), although the relative amounts are unknown. Voyager observations of these low-energy cosmic rays in the outer heliosphere during quiet solar conditions, revealed $\sim 26$-day recurrent enhancements (Decker et al., 1995). This suggests that corotating interaction regions accelerate charged particles in the inner heliosphere which convect to the outer heliosphere. These seed particles eventually interact with the termination shock of the solar wind and are further accelerated to anomalous cosmic ray energies. We have shown that the intensities of these seed particles may be high enough to account for the observed levels of anomalous cosmic rays (Giacalone et al., 1997; see also Jokipii and Giacalone, 1998). Below we further suggest that energetic particles observed by Voyager beyond about $10 \mathrm{AU}$ are most likely composed of accelerated interstellar pickup ions. We therefore suggest that the acceleration of freshly-ionized pickup ions to anomalous cosmic rays, spanning some 5 orders of magnitude in energy, occurs via a two-stage process.

\subsection{The OXYGEN CHARge State AND INNER Heliosphere PRE-ACCELERATION}

It has been pointed out (R. Mewaldt and L. Fisk, private communication) that such a two-stage mechanism, in which the overall time scale for accelerating pickup ions to anomalous cosmic-ray energies is longer than if it took place locally at the termination shock, may lead to additional electron stripping of heavier nuclei, such as oxygen. The observations of multiply-charged anomalous oxygen by Mewaldt et al. (1996b) represent an additional constraint.

To see if the inner-heliosphere pre-acceleration picture is consistent with the observations of multiply-charged cosmic rays, we used the same model as Jokipii 


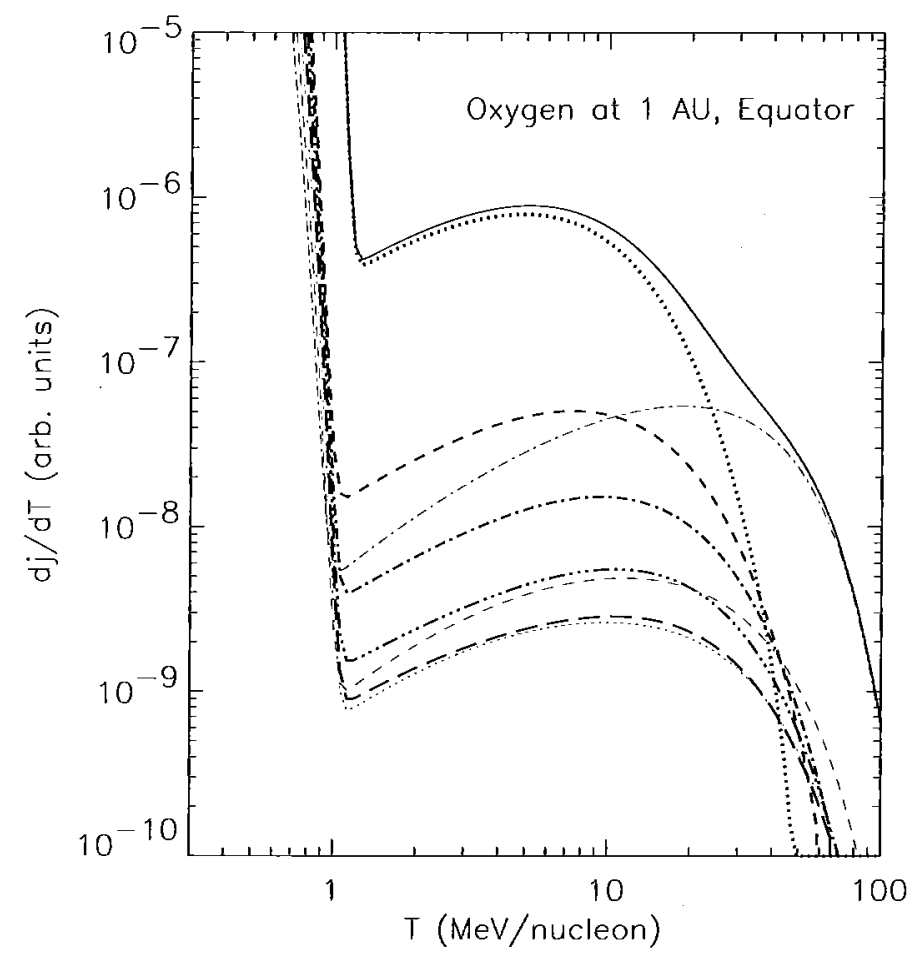

Figure 21. Computed spectra of the charge states of anomalous oxygen as a function of energy at $1 \mathrm{AU}$. The solid curve is the sum of the eight charge states. The $q=1$ (singly charged) charge state dominates at low energies (dotted curve), and the $q=8$ state dominates at high energies (dot-dashed curve). The other charge states are not easily ordered.

(1996), except with a source in the inner heliosphere, as described above. That is, we ran the same code as used by Jokipii (1996), including all eight non-zero oxygen charge states and a probability of further electron loss, with an inner-heliosphere source of pre-accelerated, single-ionized oxygen. The results of the simulation are shown in Figure 21. Clearly, the general form of the spectra are similar to those published by Jokipii (1996), confirming that the inner-heliosphere source is consistent with observations of multiply-charged oxygen. The rapid rise of the spectra below $\approx 1 \mathrm{MeV} /$ nuc is due to the assumed source at low energies.

\subsection{THE ACCELERATION OF PICKUP IONS IN THE INNER HELIOSPHERE}

We have recently shown that the acceleration of pickup ions is strongly favored at the reverse shock (Giacalone and Jokipii, 1997; see also Jokipii and Giacalone, 1998) of a shock pair associated with a corotating interaction region. This is because freshly-ionized pickup ions that interact with the reverse shock were ionized in the fast solar wind and are more energetic than those that are ionized in the slow wind. 
Consider the interaction of freshly ionized pickup ions with a propagating reverse shock. If the speed $U \sec \theta_{B n} b^{-1 / 2}$ ( $U$ is the flow speed upstream of the reverse shock relative to the shock frame, $\theta_{B n}$ is the acute angle between the shock normal and upstream magnetic field, and $b$ is the jump in field strength across the shock) is comparable to the speed of the pickup ions, then they will be readily accelerated to higher energies (e.g. Giacalone and Jokipii, 1996, 1997). It is straightforward to show that this criterion leads to

$$
\cos \theta_{B n}(r) b^{1 / 2}(r)=\frac{s}{s-1}\left(1-\frac{U_{\text {fast }}}{U_{\text {int }}}\right)
$$

where $r$ is heliocentric distance, $s$ is the density compression across the reverse shock, $U_{\text {fast }}$ and $U_{\text {int }}$ are the solar wind speeds measured relative to the sun on each side of the reverse shock, respectively.

Using the properties of the Parker-Archimedean spiral to obtain a functional form for $\theta_{B n}(r)$ and $b(r)$ and the typical values $s=3, U_{\text {fast }}=800 \mathrm{~km} / \mathrm{s}$, and $U_{\text {int }}=600 \mathrm{~km} / \mathrm{s}$, we find that $r \approx 9 \mathrm{AU}$. This means that pickup ions can be

readily accelerated by propagating reverse shocks out to about 9 AU. Beyond this, the geometry of the magnetic field is not favorable for the acceleration of freshly-ionized pickup ions and the injection diminishes.

Since reverse shocks typically form between 2-3 AU, pickup ions should be accelerated in the region 2-9 AU. Therefore, we do not expect to see accelerated pickup ions inside about 2 AU. Moreover, since interstellar hydrogen and oxygen have large ionization distances, the peak densities of these pickup ions are at some 4-8 AU, indicating that we do not expect to observe accelerated pickup protons and oxygen inside $4 \mathrm{AU}$. On the other hand, interstellar helium penetrates inside the Earth's orbit and the peak density of pickup helium is about $1 \mathrm{AU}$. We conclude that energetic protons and oxygen, associated with reverse shocks at distances larger than about $4 \mathrm{AU}$, should be composed mainly of interstellar pickup ions. On the other hand, energetic He should be mostly interstellar pickup ions beyond about 2 AU.

\subsection{DiSCUSSION AND SUMMARY}

We have discussed the possibility that anomalous cosmic rays are formed via a two-stage acceleration process of interstellar pickup ions. The first step occurs in the inner heliosphere while the final step occurs at the termination shock. We have shown that this picture, which we have described previously (c.f. Jokipii and Giacalone, 1998), is also consistent with the observations of multiply-charged anomalous oxygen. Additionally, we find that during quiet solar conditions, the reverse shock associated with corotating interaction regions can readily accelerate pickup ions out to nearly $10 \mathrm{AU}$. Consequently, we suggest that energetic nuclei in the outer heliosphere are mostly composed of accelerated interstellar pickup ions completing the first step of the two-stage acceleration mechanism. 


\section{The Ionic Charge Composition of Anomalous Cosmic Rays}

B. Klecker, R.A. Mewaldt, M. Oetliker, R.S. Selesnick, and J.R. Jokipii

\subsection{INTRODUCTION}

It is now generally accepted that the source of ACRs is interstellar neutral particles, which become ionized by either solar UV or by charge exchange with solar wind protons, and are then convected with the solar wind and accelerated in the outer heliosphere as originally proposed by Fisk et al. (1974). The unique prediction of this theory was that ACR ions should be singly charged, in contrast to solar energetic particles (SEP) or galactic cosmic rays (GCR), which have high charge states. The hypothesis of an interstellar source was supported by a number of indirect methods to determine the ionic charge based on modulation arguments (see e.g. Klecker, 1995 for a recent review). Only recently was the theory fully confirmed by a more direct measurement of the ionic charge of ACRs using the Earth's magnetic field as a magnetic spectrometer: at energies of $\approx 10 \mathrm{MeV} /$ nuc a mean ACR oxygen charge of $0.9\left(+0.3 /-0.2\right.$, Adams et al., 1991), $\mathrm{O}^{2+} / \mathrm{O} \$ 15 \%$ (Klecker et al., 1995), and a large number of $\mathrm{N}^{+}$and $\mathrm{Ne}^{+}$ions at low magnetic latitudes (Klecker et al., 1995) have been found. More recently, multiply charged ACR oxygen has been discovered at energies $\gtrsim 16 \mathrm{MeV} / \mathrm{nuc}$ (Mewaldt et al., 1996b) and it has been shown that the abundance is roughly consistent with charge exchange cross sections and acceleration at the termination shock (Jokipii, 1996).

In this report we summarize the present status of the ACR ionic charge analysis. With the now much improved statistics of more than 3 years of SAMPEX data, the determination of the ionic charge composition has been extended to the energy range 8-100 MeV/nuc (for oxygen) and to the less abundant elements nitrogen and neon.

\subsection{OBSERVATIONS OF THE IONIC CHARGE COMPOSITION OF ACR IONS}

The observations summarized here were made with instruments on board the SAMPEX satellite, which was launched into a $510 \times 675 \mathrm{~km}, 82^{\circ}$ inclination Earth orbit on July 3, 1993 (Baker et al., 1993). The ACR fluxes were measured with two sensors, the Heavy Ion Large Telescope (HILT, Klecker et al., 1993) and the Mass Spectrometer Telescope (MAST, Cook et al., 1993) which provide very large sensitivities of $60 \mathrm{~cm}^{2} \mathrm{sr}$ and $\sim 14 \mathrm{~cm}^{2} \mathrm{sr}$, respectively, and cover the wide energy range from $\sim 8-160 \mathrm{MeV} /$ nuc (for oxygen) required for precise ACR studies. With the recent approach to solar minimum, ACR fluxes at Earth began rising significantly in early 1992 (Mewaldt et $a l ., 1993$ ) with a gradual increase until 1996, providing sufficient counting statistics for ionic charge analysis.

The analysis of the ionic charge of ACR ions is based on the limiting ("cutoff") magnetic rigidity, or momentum per unit charge, $P_{c}$, for which a particle can reach 
HILT / SAMPEX Quiet Time Data
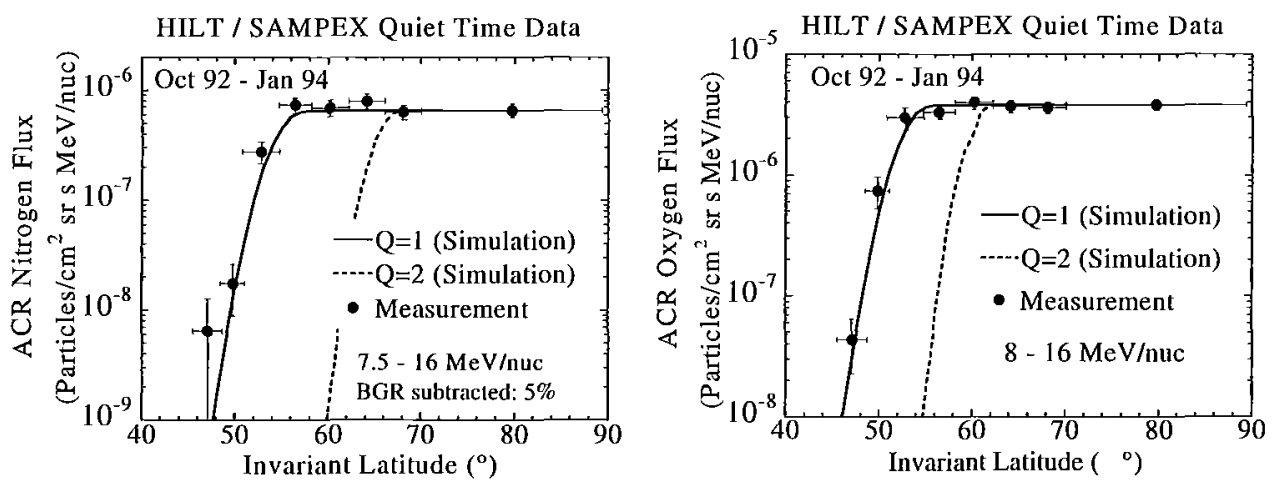

Figure 22. Flux of ACR nitrogen (left) and oxygen nuclei (right) in the energy range $8-16 \mathrm{MeV} / \mathrm{nuc}$ as a function of invariant latitude. The solid and dotted lines show the expected flux for singly and doubly ionized $\mathrm{N}$ and $\mathrm{O}$, respectively.

the detector for a specific incident trajectory. If a non-trapped particle reaches the sensor, then the upper limit of the particle's ionization state, $q_{u}$, is given by

$$
q_{u}=(m v) /\left(e P_{c}\right)
$$

where $m$ is the particle mass, $v$ is the velocity, and $e$ is the electron charge. Since $m$ and $v$ are determined by the measurement, the charge state upper limit, $q_{u}$, for each ion can be found by calculating the cutoff rigidity for its trajectory, and then using equation (12). The ionic charge composition can be derived from the measured flux as a function of invariant latitude (). This can be accomplished by comparing the measured intensity- profiles with calculations for different ionic charge states (see e.g. Klecker et al., 1995; Mewaldt et al., 1996b). Figure 22 shows, as an example of this method, the measured intensity-profiles for ACR nitrogen and oxygen in the energy range $\sim 8-16 \mathrm{MeV} /$ nuc, together with the expected flux variation for singly and doubly ionized nitrogen and oxygen, computed with a relation between cutoff rigidity and invariant latitude derived from trajectory calculations. Figure 22 demonstrates that in the energy range $8-16 \mathrm{MeV} /$ nuc the flux profile is consistent with ACR nitrogen and oxygen being predominantly singly ionized.

With another method introduced recently, instead of invariant latitude, the coordinate $q_{u}$, the upper limit of the ionic charge, $q$, is used for binning the data. In this representation the contributions from individual charge states, $q_{i}$, to the total intensity are step functions in $q_{u}$. The ionic charge composition can be derived with a least squares fit procedure from a superposition of step functions for the individual charge states $q_{i}$ (Oetliker et al., 1997b; Selesnick et al., 1997). As an example, the measured charge profiles of oxygen in 5 energy bands between 8 and $26 \mathrm{MeV} /$ nuc are shown in Figure 23 (from Klecker et al., 1997). In this analysis four contributions were used for the fit of the charge distribution, $q=+1,+2,+3$, and $\geq 4$. Figure 23 demonstrates that the ionic charge composition of ACR oxy- 


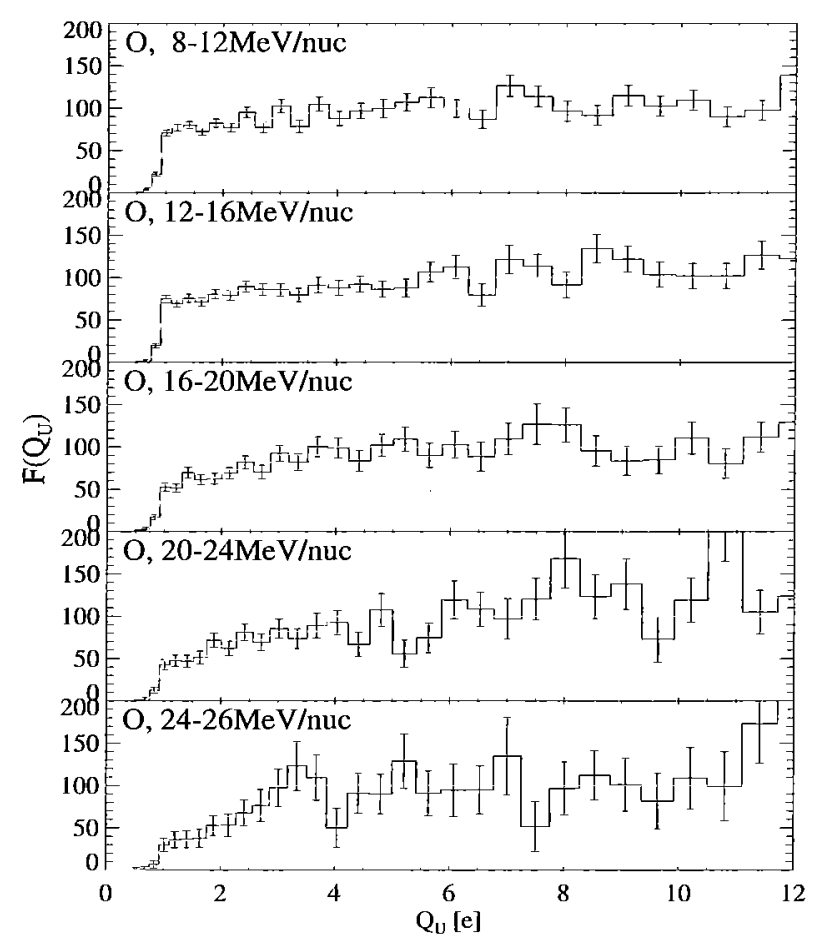

Figure 23. Ionic charge profiles with fit for 5 energy ranges of ACR oxygen between 8 and $26 \mathrm{MeV} / \mathrm{nuc}$ (from Klecker et al., 1997).

gen in this energy range is continuously changing with energy, with singly ionized ions dominating at 8-12 MeV/nuc and higher charge states dominating above $20 \mathrm{MeV} / \mathrm{nuc}$.

The energy dependence of the ionic charge composition of ACR N, O, and Ne is shown quantitatively in Figure 24. Here we combined measurements of the charge composition in the energy range 8-100 MeV/nuc reported by Klecker et al. (1997) and Selesnick et al. (1997) and show the abundances of singly ionized N, O, and $\mathrm{Ne}$ relative to the sum of charge states $1-3$ as a function of energy. It is evident from Figure 24 that not only ACR O, but also $\mathrm{N}$ and Ne at energies $\lesssim 20 \mathrm{MeV} / \mathrm{nuc}$ are predominantly singly ionized. Above $\sim 20 \mathrm{MeV} /$ nuc the abundances of singly ionized ions fall off sharply and multiply charged ions become dominant. Figure 24 also suggests that the energy of the sharp drop, e.g. the energy where the abundances of singly charged ACR ions drop to $50 \%\left(E_{50 \%}\right)$ is mass dependent, as was also pointed out by Selesnick et al. (1997). We estimated $E_{50 \%}$ with a logarithmic fit of the abundances in the energy range of the sharp abundance drop ( $Z \gtrsim 13$ (10) $\mathrm{MeV} / \mathrm{nuc}$ for $\mathrm{N}, \mathrm{O}(\mathrm{Ne})$, dashed line in Figure 24). The corresponding values of $E_{50 \%}$ are $25.5 \pm 2.5,22 \pm 2$, and $17.5 \pm 2.0$ (MeV/nuc) for $\mathrm{N}, \mathrm{O}$, and $\mathrm{Ne}$, respectively. Although the statistical uncertainty is large, the results suggest a trend with higher- 

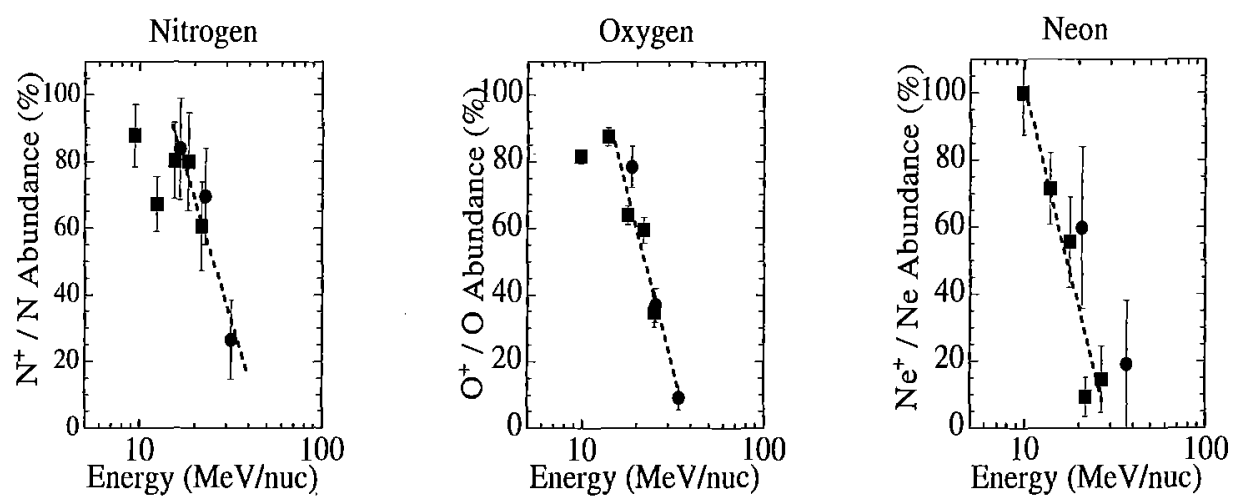

Figure 24. Relative abundance of singly ionized ACR N, O, and Ne as a function of energy (from Klecker et al., 1997 (squares), and Selesnick et al., 1997 (circles)).

mass singly-charged ACRs dropping off at lower energy per nucleon with a drop-off total energy of $\sim 350 \mathrm{MeV}$.

\subsection{DISCUSSION AND SUMMARY}

The observations of the ACR N, O, and Ne ionic charge composition with SAMPEX show that singly charged ions, originally expected for the interstellar source suggested by Fisk et al. (1974), dominate only at energies below $20 \mathrm{MeV} / \mathrm{nuc}$. At higher energies, multiply charged ions become more abundant. It has been pointed out by Jokipii (1992) and Klecker (1995) that the low abundances of multiply charged ions at $\sim 10 \mathrm{MeV} /$ nuc place important constraints on the acceleration time scales in the outer heliosphere. The acceleration process has to be sufficiently fast to compete with losses by charge exchange reactions with the ambient interstellar neutral hydrogen and by adiabatic deceleration (see also Mewaldt et al., 1996b). Figure 25 shows the time scale for stripping of $\mathrm{O}^{+}$as a function of energy per nucleon, using stripping cross sections compiled by Spjeldvik (1979), a neutral hydrogen density of $0.1 \mathrm{~cm}^{-3}$, and assuming stripping of $30 \%$ of $\mathrm{O}^{+}$. Also shown are the convection time scales for a solar wind velocity of $400 \mathrm{~km} / \mathrm{s}$. Figure 25 illustrates that most of the stripping occurs at low energies of $\sim 0.1-1 \mathrm{MeV} / \mathrm{nuc}$, as pointed out by Mewaldt $e$ t al. (1996b), and that the convection time scales are of the order of the time scales for stripping at these low energies. This provides severe constraints for pre-acceleration models because it limits the transport time scales to the outer heliosphere at these low energies.

The dominance of ions with $q>1$ at high energies implies that multiply charged ions have gained more energy during the acceleration process and explains how ACRs get accelerated to energies approaching $100 \mathrm{MeV} /$ nuc (Mewaldt et al., 1996a). This is consistent with most of the stripping occurring at low energies, and an acceleration of these multiply charged ions at the termination shock (see below). 


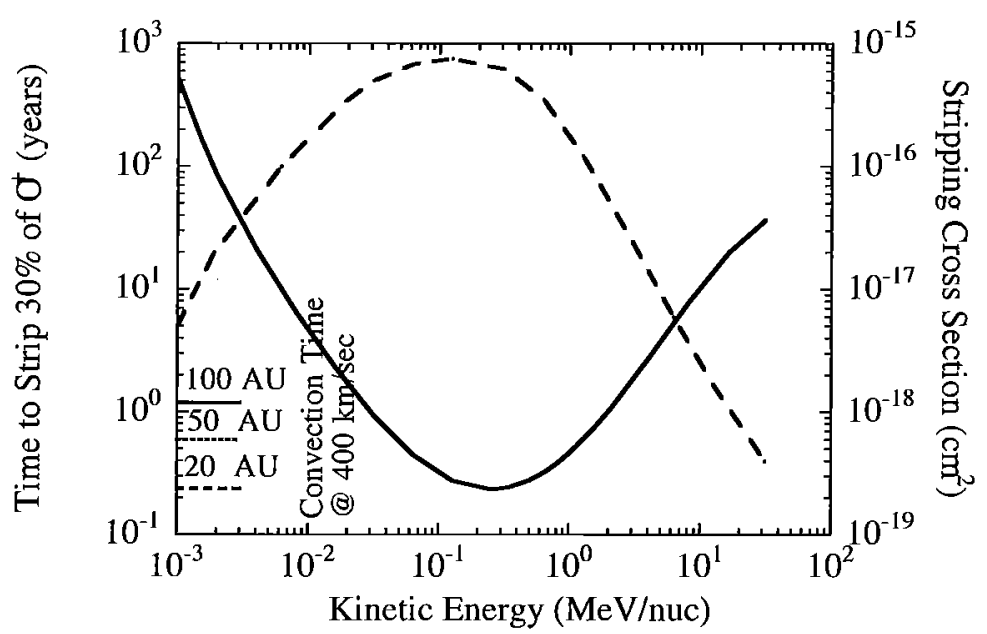

Figure 25. Charge exchange time scales for stripping $30 \%$ of $^{+}$(solid line, left scale) and stripping cross section from Spjeldvik (1979) (dashed line, right scale). Also shown are the convection time scales for a $400 \mathrm{~km} / \mathrm{s}$ solar wind.

It has been shown that acceleration at the quasi-perpendicular termination shock could also provide the necessary short time scale of the order of $\sim 1$ year (Jokipii, 1992). A recent detailed two-dimensional model calculation for the acceleration of ACR oxygen, including acceleration at the termination shock and charge exchange losses (Jokipii, 1996) is in reasonable good agreement with the observations of singly charged oxygen ions dominating below $\sim 20 \mathrm{MeV} /$ nuc. Above this energy the model also shows the dominance of the higher charge states, although with a somewhat larger mean ionic charge than observed (see also Section 7). This is possibly due to uncertainties in the oxygen charge exchange cross sections, which are not all well known.

The sharp drop of the abundance of singly ionized oxygen at $\sim 20 \mathrm{MeV} / \mathrm{nuc}$, however, is well reproduced by the model. This drop can also be qualitatively understood in terms of the characteristic energy, $E_{\max }$, the particles can gain at the termination shock:

$$
E_{\text {max }}={ }_{\max } q
$$

where $_{\max } \sim 240 \mathrm{MV}$, and $q$ is the ionic charge of the ion (e.g. Jokipii and Giacalone, 1998). Equation (13) shows that for singly charged ions the characteristic maximum energy is $240 \mathrm{MeV}$, i.e. the characteristic energy per nucleon will be ordered by mass. Thus, for ACR N, O, and Ne the highest characteristic energy per nucleon can be expected for singly ionized ACR nitrogen, the lowest for neon with oxygen being in between. The observed variation of $E_{50 \%}$, i.e. the constant value of $E_{50 \%} A(\sim 350 \mathrm{MeV}$, see above) is in good agreement with this expectation. The precise value of the energy where the abundance of singly ionized ACR ions drops off depends, however, on an interplay between the acceleration and propagation 
processes in the heliosphere. The presently available data on the ionic charge composition provide the basis for future more detailed comparisons of the ACR charge composition with model calculations.

\section{A Search for Minor Ions in Anomalous Cosmic Rays}

B. Klecker, R.A. Mewaldt, M. Oetliker, and R.A. Leske

\subsection{INTRODUCTION}

There are presently seven elements that are generally recognized as members of the ACR component: $\mathrm{H}, \mathrm{He}, \mathrm{C}, \mathrm{N}, \mathrm{O}, \mathrm{Ne}$, and Ar. All of these, except $\mathrm{C}$, have relatively high first ionization potentials, ranging from $\sim 13.6 \mathrm{eV}$ for $\mathrm{H}$ to $\sim 24 \mathrm{eV}$ for $\mathrm{He}$. It is therefore expected that a large fraction of these species are in a neutral state in the local interstellar medium (LISM). Carbon, with its lower first ionization potential ( $\sim 11 \mathrm{eV}$ ), is expected to be mostly ionized in the LISM. Recently, Reames et al. (1996) and Takashima et al. (1997) reported possible evidence for ACR sulfur at 1 AU. Sulfur has a first ionization potential of $\sim 10.4 \mathrm{eV}$, similar to $\mathrm{C}$, and somewhat higher compared to neighboring elements such as $\mathrm{Mg}(\sim 7.6 \mathrm{eV})$ and Si $(\sim 8.1 \mathrm{eV})$. In an effort to verify these new $\mathrm{S}$ results, Stone and Cummings (1997) examined Voyager 1 and 2 data from the outer heliosphere. They found enhanced abundances of $\mathrm{Si}, \mathrm{S}$, and $\mathrm{Fe}$, which they attributed to anomalous cosmic rays. However, the relative abundance of $S$ they observed was much less than that reported at $1 \mathrm{AU}$ (see also Table VIII) suggesting that there may be an additional source of low-energy $S$ or that none of the $S$ is of ACR origin.

The present situation with S may be similar to that with ACR C. Beginning with the discovery of ACR ions more than 20 years ago, the quiet time analysis of low energy interplanetary spectra of $\mathrm{C}$ and $\mathrm{O}$ in the inner heliosphere generally showed $\mathrm{C} / \mathrm{O}$ ratios of $\sim 5-15 \%$ (Oetliker et al., 1997b, and references therein), much higher than expected from an interstellar source. The first convincing evidence for ACR $\mathrm{C}$ was presented by Cummings and Stone (1988) based on Voyager data from $\sim 20 \mathrm{AU}$ during the 1987 solar minimum. The observed $\mathrm{C} / \mathrm{O}$ ratio was $\sim 0.01$, consistent with the interpretation that only a small fraction of $\mathrm{C}$ is neutral in the LISM. Using Ulysses data, Geiss et al. (1995) reported evidence for an additional "inner source" of C, N, and O: they observed an enhanced abundance of C among pickup ions at distances $\lesssim 4 \mathrm{AU}$, which they attributed to contributions from interstellar dust. However, a quantitative estimate showed (Geiss et al., 1996) that the total production of $\mathrm{C}^{+}$and $\mathrm{O}^{+}$from this inner source is only of the order of $\sim 0.1 \%$ as compared to the total production of $\mathrm{O}^{+}$from the interstellar gas and does not contribute significantly to $\mathrm{O}$ (or $\mathrm{N}$ ) in the ACRs. However, its contribution to ACR C may not be negligible and could be of the order of $\sim 10 \%$. In a recent SAMPEX analysis, Mewaldt et al. (1996a) and Oetliker et al. (1997b) used the Earth's magnetic field as a rigidity spectrometer to filter out high charge states 
from the low-energy quiet-time spectra and obtain a "pure" sample of ACRs. They found ACR C/O ratios of 0.014 0.009 (17-42 MeV/nuc) and $0.021 \pm 0.009$ (8-20 MeV/nuc), respectively, consistent with the observations by Voyager in the outer solar system. These low abundances indicate that most low-energy $\mathrm{C}$ at $1 \mathrm{AU}$ in interplanetary space is not singly charged, as expected for ACRs, and that additional sources may contribute to the quiet-time fluxes at $1 \mathrm{AU}$.

In this section we report preliminary results of an extension of the SAMPEX geomagnetic filter approach, including data from mid-1992 to early 1996, as measured with the HILT (Klecker et al., 1993) and MAST (Cook et al., 1993) instruments on SAMPEX. The advantage of this approach is that particles of galactic origin, which are essentially fully stripped, or of solar and interplanetary origin (e.g. SEP, CME), which have high charge states characteristic of coronal temperatures of about $2 \cdot 10^{6} \mathrm{~K}$ (Luhn et al., 1984, Oetliker et al., 1997a, and references therein), are filtered out, leaving a pure sample of ions with low charge states (e.g., $q=+1,+2$, etc).

\subsection{OBSERVATIONS}

SAMPEX is in a near polar orbit (82 inclination) that cuts across geomagnetic cutoffs from $\sim 0$ to $\sim 15 \mathrm{GV}$ four times per orbit. Using particle trajectory calculations and empirical geomagnetic cutoff relations derived from fully-stripped galactic cosmic rays and solar energetic particles (see Klecker et al., 1995; Mewaldt et al., 1996a), it is possible to filter out GCR and SEP nuclei as well as trapped ACRs (Selesnick et al., 1995) to obtain a "pure" sample of ACRs. HILT covers the low energy range from $\sim 8$ to $28 \mathrm{MeV} /$ nuc (for O), while MAST covers the higher energy range, varying from $14-32 \mathrm{MeV} / \mathrm{nuc}$ for $\mathrm{C}$ to $29-60 \mathrm{MeV} / \mathrm{nuc}$ for $\mathrm{Fe}$, in this analysis. Relative abundances of $\mathrm{C}, \mathrm{O}, \mathrm{Ne}, \mathrm{Mg}, \mathrm{Si}, \mathrm{S}, \mathrm{Ar}$, and $\mathrm{Fe}$ with low charge states have been obtained over a large energy range (Table VII).

At low energies, using HILT data during the time period September 1992 to July 1995, and following the approach of Oetliker et al. (1997b), relative abundances of ions with low charge states in the energy range $\sim 8-20(\mathrm{C}-\mathrm{Ne})$ and $\sim 10$ $20 \mathrm{MeV} / \mathrm{nuc}(\mathrm{Mg}-\mathrm{Fe})$ have been derived from ionic charge analysis. At higher energies, the geomagnetic filter approach has been used to discriminate against high charge states by selecting data from mid latitudes of $\sim 52^{\circ}$ to $65^{\circ}$, where highly charged ions do not have access (Mewaldt et al., 1996a). Figure 26 shows that in the MAST energy range for $\mathrm{Z}>10$ only $1 \mathrm{Mg}$ and $1 \mathrm{Si}$ count have been observed. The results on elemental composition are summarized in Table VII. The abundances reported recently from interplanetary measurements at 1 AU (Reames et al., 1996; Takashima et al., 1997) and in the outer heliosphere (Stone and Cummings, 1997) are summarized in Table VIII.

The abundances of $\mathrm{Si}$ and Ar with $q<4$ are compatible with the observations in the outer heliosphere. However, somewhat surprisingly, the upper limit for singly charged Ar is significantly smaller than both the interplanetary measurements in 


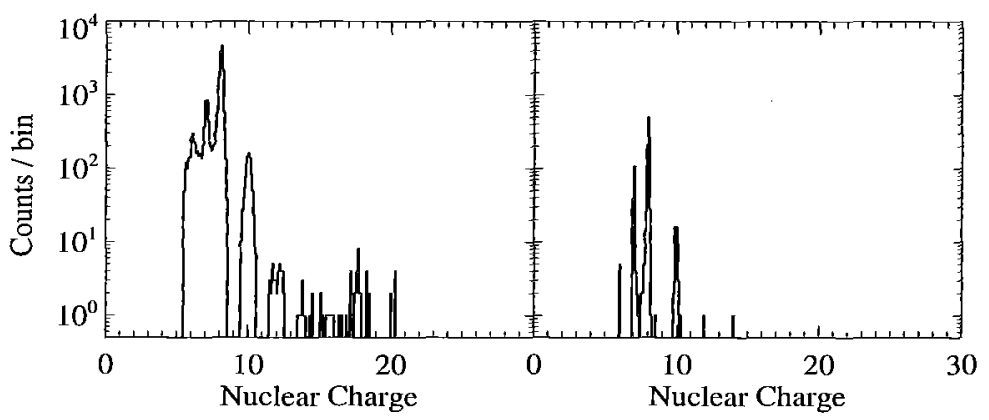

Figure 26. Histogram of events with low charge states from HUL (left) and MAST (right).

Table VII

Summary of elemental abundances derived with the geomagnetic filter approach at intermediate latitudes and from trapped particles at low latitudes ( $1 \sigma$ errors are given in parentheses)

\begin{tabular}{lc|lll|ll|l}
\hline Element & $\mathrm{Z}$ & $\begin{array}{l}\text { Energy } \\
(\mathrm{MeV} / \mathrm{nuc})\end{array}$ & $\begin{array}{l}\text { HILT } \\
(q=1)\end{array}$ & $\begin{array}{l}\text { HILT } \\
(q<4)\end{array}$ & $\begin{array}{l}\text { Energy } \\
(\mathrm{MeV} / \mathrm{nuc})\end{array}$ & $\begin{array}{l}\text { MAST } \\
\text { Filtered }\end{array}$ & $\begin{array}{l}\text { MAST } \\
\text { Trapped }\end{array}$ \\
\hline $\mathrm{C}$ & 6 & $7-20$ & $0.9(0.5)$ & $2.7(1.1)$ & $14-32$ & $0.7(0.4)$ & $0.08(0.03)$ \\
$\mathrm{O}$ & 8 & $8-20$ & 70 & 86 & $17-42$ & 100 & 100 \\
$\mathrm{Ne}$ & 10 & $9-20$ & $3.5(0.3)$ & $4.5(0.6)$ & $19-46$ & $6.3(1.1)$ & $3.0(0.25)$ \\
$\mathrm{Mg}$ & 12 & $9-20$ & $0.07(0.03)$ & $0.1(0.03)$ & $21-51$ & $<0.7$ & $<0.05$ \\
$\mathrm{Si}$ & 14 & $10-20$ & $<0.03$ & $<0.07$ & $22-56$ & $<0.7$ & $<0.06$ \\
$\mathrm{~S}$ & 16 & $10-20$ & $<0.03$ & $<0.10$ & $24-60$ & $<0.5$ & $<0.09$ \\
$\mathrm{Ar}$ & 18 & $10-20$ & $<0.02$ & $0.10(0.03)$ & $25-60$ & $<0.6$ & $<0.11$ \\
$\mathrm{Fe}$ & 26 & $10-20$ & $<0.02$ & $<0.02$ & $29-60$ & $<0.7$ & $<0.23$ \\
\hline
\end{tabular}

the outer heliosphere and at $1 \mathrm{AU}$. The upper limit for Fe with $q<4$ is also significantly smaller than the abundance observed at Voyager. The upper limit of $\mathrm{S}^{+} / \mathrm{O}$ is compatible with the abundance in the outer heliosphere. However, the interplanetary S/O ratio in the outer heliosphere is significantly smaller than at $1 \mathrm{AU}$, as reported by Stone and Cummings (1997).

We have also searched for evidence of ACRs with $Z>10$ in the radiation belt at low latitudes ( $\sim \sim 2)$ composed of trapped ACRs (see, e.g., Selesnick et al., 1995). Among more than 13,000 trapped C, N, O, and Ne nuclei observed by MAST there were no nuclei heavier than Ne (see Table VII). This places limits on the interplanetary abundance of singly or doubly charged ACRs with $Z>10$, but they are difficult to quantify because of uncertainties in the trapping process. Note, however, that if heavy ACR nuclei have charge to mass ratios of $A / q \lesssim 12$, they are not likely to be trapped (see discussion below and Selesnick et al., 1995). 
Table VIII

Summary of elemental abundances from quiet time measurements in interplanetary space. The data are taken from Reames et al. (1996) ${ }^{1}$, Takashima et al. $(1997)^{2}$, and Stone and Cummings $(1997)^{3}$

\begin{tabular}{ll|lll|l}
\hline Element & $\mathrm{Z}$ & $\begin{array}{l}\text { Geotail }^{2} \\
\sim 10 \\
(\mathrm{MeV} / \mathrm{nuc})\end{array}$ & $\begin{array}{l}\text { Wind }^{1} \\
\sim 10 \\
(\mathrm{MeV} / \mathrm{nuc})\end{array}$ & $\begin{array}{l}\text { Geotail }^{2} \\
(\mathrm{MeV} / \mathrm{nuc})\end{array}$ & $\begin{array}{l}\text { Voyager }^{3} \\
\sim 6-20 \\
(\mathrm{MeV} / \mathrm{nuc})\end{array}$ \\
\hline $\mathrm{C}$ & 6 & 9 & 2.9 & 18 & $\sim 2.0$ \\
$\mathrm{O}$ & 8 & 100 & 100 & 100 & 100 \\
$\mathrm{Ne}$ & 10 & 5 & 6 & 4.4 & 4.8 \\
$\mathrm{Mg}$ & 12 & & 0.23 & & \\
$\mathrm{Si}$ & 14 & 0.25 & & 1.0 & $\sim 0.07$ \\
$\mathrm{~S}$ & 16 & 0.10 & & 0.24 & $\sim 0.02$ \\
$\mathrm{Ar}$ & 18 & & 0.25 & & $\sim 0.15$ \\
$\mathrm{Fe}$ & 26 & & & 0.83 & $\sim 0.07$ \\
\hline
\end{tabular}

\subsection{DISCUSSION}

The geomagnetic filter approach to discriminate against high charge states provides a valuable tool to derive the composition of a "pure" ACR sample in the inner heliosphere. It has been demonstrated that most of the low energy quiet time $\mathrm{C}$ observed in interplanetary space at $\sim 1 \mathrm{AU}$ is not of ACR origin. However, the observed $\mathrm{C} / \mathrm{O}$ ratio of $\sim 1 \%$ for low charge states is compatible with the observations in the outer heliosphere where contamination by solar / interplanetary particles is apparently less significant. Table VII shows that abundances derived for low charge states $(q<4)$ at $\sim 10 \mathrm{MeV} /$ nuc are in general consistent with the abundances observed in the outer heliosphere, with the exception of Fe, where low charge states are significantly less abundant. This also seems to be true for singly ionized $\mathrm{Si}$ and $\mathrm{Ar}$ with energies $>10 \mathrm{MeV} /$ nuc.

The interplanetary measurements at $\sim 1 \mathrm{AU}$ and in the outer heliosphere are not consistent for $\mathrm{C}$ and $\mathrm{S}$. Although there is no direct evidence from the $\mathrm{S}$ ionic charge measurement, this suggests that at $\sim 1 \mathrm{AU}$ a significant fraction of $\mathrm{S}$ (as most of C) may be from a highly charged solar or interplanetary component. The low energy ( $<10 \mathrm{MeV} / \mathrm{nuc}$ ) S/C ratio of $\sim 0.015$ as observed at $1 \mathrm{AU}$ (Takashima et al., 1997) is, for instance, only a factor of $\sim 2$ smaller than coronal hole type solar wind composition ( 0.031, Shafer et al., 1993) and a factor of $\sim 4-5$ smaller than typical in-ecliptic solar wind $(\sim 0.053-0.07$, Geiss et al., 1994) or SEP composition ( 0.08, Breneman and Stone, 1985).

If low energy $\mathrm{Si}$ and $\mathrm{Fe}$ as measured by Voyager are of $\mathrm{ACR}$ origin, then the low abundances of $\mathrm{Si}^{+}$and $\mathrm{Fe}^{+}$at $1 \mathrm{AU}$ (as of $\mathrm{Ar}^{+}$) could qualitatively be understood in terms of the acceleration process: the maximum energy particles of charge $q$ can gain at the termination shock is $\sim 240 q(\mathrm{MeV})$ (Jokipii and Giacalone, 1998). 
This is consistent with the observation that the energy per nucleon above which multiply charged ions dominate is decreasing with mass (Selesnick et al., 1997; Section 8). Using the value of $350 \mathrm{MeV}$ for the energy where the abundances of singly charged ions drop to $50 \%$, as derived in Section 8 for ACR N, O, and $\mathrm{Ne}$, the corresponding energy per nucleon for $\mathrm{Si}, \mathrm{Ar}$, and $\mathrm{Fe}$ would be $12.5,9.7$, and $6.3 \mathrm{MeV} /$ nuc. Therefore, in the energy range $\geq 10 \mathrm{MeV} /$ nuc not many singly ionized Ar and Fe ions would be expected. Furthermore, the abundance of multiply charged ions will also depend on the charge exchange cross sections. For example, the cross sections for stripping 1 or 2 electrons from $\mathrm{Ar}^{+}$at $2 \mathrm{MeV}$, i.e. near the energy range of the maximum stripping cross section, are a factor of $\sim 2.5$ larger than the coiresponding cross sections of oxygen (Lo et al., 1971). For Fe the corresponding cross sections are a factor of $\sim 2$ smaller than for oxygen, and do not provide an explanation for increased losses. Thus, the question of the source of low energy S, Si, and Fe needs further investigation. Interplanetary measurements with improved collecting power or closer to the termination shock, in combination with ionic charge analysis and model calculations for minor ions will help to improve our understanding of rare ACR species.

\section{Summary}

As we conclude this chapter it is appropriate to revisit the "Top Ten Questions in Anomalous Cosmic Ray Studies" listed in Table I, in order to assess in which areas there has been progress.

Questions 1 and 2 concern possible additional species and/or sources of ACRs. The search for rare ACR ions in Section 9 demonstrates the power of the geomagnetic technique for addressing this question; this approach can separate partiallyionized from fully-stripped ions. This work provides confirming evidence that there are at least two sources of low energy carbon ions at $1 \mathrm{AU}$ in addition to the lowenergy tail of the modulated GCR component. There is apparently an interstellar neutral source with $\mathrm{C} / \mathrm{O} \sim 0.01-0.02$ that is consistent with Voyager observations, and also an additional source of ions of unknown origin that appear to be dominated by intermediate charge states $(2 \leq q \leq 5)$.

Section 9 also indicates that the low-energy sulfur component reported by Wind and Geotail (Reames et al., 1996; Takashima et al., 1997) may partly have an origin other than the neutral ISM, since apparently little of these S ions are singly-charged. In addition, there appears to be low-energy $\mathrm{Mg}, \mathrm{Si}$, and $\mathrm{Ar}$ ions that are neither fully-stripped nor singly-charged. The Ar is likely of interstellar neutral origin that (at energies $>8 \mathrm{MeV} / \mathrm{nuc}$ ) has been stripped to charge states of $q>1$ during the ACR acceleration process (see below). The origin of the partially-ionized $\mathrm{Mg}$ and $\mathrm{Si}$ ions remains unclear, but it is evident that this field will continue to be one of vigorous investigation over the coming years. 
Questions 3, 4, and 5 are concerned with the injection of pickup ions into the acceleration process, with the observed mass dependent acceleration efficiency and with the possible pre-acceleration of pickup ions in the heliosphere. It has been shown that acceleration in the inner heliosphere by CIRs and in the outer heliosphere by stochastic acceleration, in particular in corotating merged interaction regions, could significantly contribute to the particle population finally accelerated to ACR energies at the termination shock. It has also been demonstrated that the increase of the acceleration efficiency with mass could be reproduced in the mass range 1 to 4 , at least qualitatively. However, a corresponding increase at higher masses could not be shown yet. As the next step, the pre-acceleration models including representative elements (e.g. $\mathrm{H}^{+}, \mathrm{He}^{+}, \mathrm{O}^{+}$) have to be combined with models for acceleration at the termination shock and propagation in the heliosphere to fully understand the processes governing ACR injection, acceleration and propagation in the heliosphere (see below).

It was not expected that new measurements of ACR gradients and energy spectra would lead immediately to improved interplanetary diffusion coefficients and other transport parameters (Question 6), but the spacecraft data sets that are compiled in Section 2 represent a necessary first step towards this goal by providing bench mark data sets against which new models of cosmic ray transport in the heliosphere will be measured. The modeling efforts in Section 3 manage to fit the spectra of $\mathrm{H}$ and $\mathrm{He}$ individually, but do not yet provide a self-consistent fit to both ACR H and He. However, this model is being actively pursued and improved results have already been reported (C. D. Steenberg, private communication). In addition, new data continue to be added to the ACR archive at ISSI.

Although we are only beginning to realize the implications of multiply-charged ACRs (Questions 7 and 8), the report in Section 8 provides clear evidence that multiply-charged ACRs are common to other ACR species besides oxygen, and it suggests a pattern to the energy range where they predominate. Based on SAMPEX data for $\mathrm{N}, \mathrm{O}$, and $\mathrm{Ne}$, it appears that singly-charged ACRs dominate below a total kinetic energy of $\sim 350 \mathrm{MeV}$, while multiply-charged ACRs dominate at higher energies. This pattern appears to be consistent with Jokipii's model of diffusive shock-drift acceleration, in which the spectra of accelerated species are expected to steepen significantly above a characteristic energy of $\sim 240 q \mathrm{MeV}$ (e.g., Jokipii, 1996).

In their review, Cummings and Stone (1998) provide evidence that the spectrum of accelerated ions observed as Voyager approaches the termination shock has a spectral index of -1.3 , suggesting that the solar wind termination shock may be a weak shock, with a strength $s$ of $<2.8$. Although none of the studies presented in this chapter address this observation specifically, during the discussions at the workshop a second possible interpretation was suggested. In the diffusive shock-drift acceleration model, the accelerated ions gain energy largely by drifting towards the poles of the heliosphere (during the current $q A>0$ phase of the solar cycle), suggesting that the highest energy particles end up in the polar 
regions. Thus, if the potential energy gained by drift is indeed significant, one might expect the accelerated spectra in the polar regions to be harder than predicted for a given shock strength, while at lower latitudes the spectra near the shock would be softer than expected from simple shock-acceleration theory (the Voyagers are both located below $35^{\circ}$ latitude). It should be possible to test this suggestion with multi-dimensional acceleration and transport models.

According to the latest predictions, Voyager 1 may cross the termination shock sometime before 2003 (e.g., Cummings and Stone, 1998). With the wealth of data now available from present solar minimum, it appears quite reasonable to expect that extensions of present models will have predictions available from a reasonably complete and self-consistent ACR acceleration/transport model (Question 10) in time for direct in situ testing by Voyager 1 . It will be an additional challenge to ensure that such models are also consistent with the well-documented behavior of galactic cosmic rays (see also the Chapter of Working Group 1).

\section{References}

Achterberg, A., Blandford, R.D. and Reynolds, S.P.: 1994, 'Evidence for enhanced MHD turbulence outside sharp rimmed supernova remnants', Astron. Astrophys. 281, 220-230.

Adams, J.H., Jr., Garcia-Munoz, M., Grigorov, N.L., Klecker, B., Kondratyeva, M.A., Mason, G.M., McGuire, R.E., Mewaldt, R.A., Panasyuk, M.I., Tretyakova, Ch.A., Tylka, A.J. and Zhuravlev, D.A.: 1991, 'The charge state of the anomalous component of cosmic rays', Astrophys. J. Lett. 375, L45-L48.

Baker, D.N., Mason, G.M., Figueroa, O., Colon, G., Watzin, J.G. and Aleman, R.M.: 1993, 'An overview of the solar, anomalous and magnetospheric particle explorer (SAMPEX) mission', IEEE Trans. Geosci. Rem. Sensing 31, 531-541.

Baring, M.G., Ogilvie, K.W., Ellison, D.C. and Forsythe, R.J.: 1997, 'Acceleration of solar wind ions by nearby interplanetary shocks: comparison of Monte Carlo simulations with Ulysses observations', Astrophys. J. 476, 889-902.

Bieber, J.W., Burger, R.A. and Matthaeus, W.H.: 1995, 'The diffusion tensor throughout the heliosphere', Proc. $24^{\text {th }}$ Int. Cosmic Ray Conf. (Rome) 4, 694-697.

Biswas, S., Durgaprasad, N., Mitra, B. and Dutta, A.: 1993: 'Anuradha and low-energy cosmic rays', Space Sci. Rev. 62, 3-65.

Bogdan, T.J., Lee, M.A. and Schneider, P.: 1991, 'Coupled quasi-linear wave damping and stochastic acceleration of pickup ions in the solar wind', J. Geophys. Res. 96, 161-178.

Breneman, H.H. and Stone, E.C.: 1985, 'Solar coronal and photospheric abundances from solar energetic particle measurements', Astrophys. J. Lett. 299, L57-L61.

Burlaga, L.F., Ness, N.F. and Belcher, J.W.: 1997, 'The radial evolution of corotating merged interaction regions and flows between $\approx 14 \mathrm{AU}$ and $\approx 43 \mathrm{AU}$ ', J. Geophys. Res. 102, 4661-4672.

Cook, W.R., Cummings, A.C., Cummings, J.R., Garrard, T.L., Kecman, B., Mewaldt, R.A., Selesnick, R.S., Stone, E.C. and von Rosenvinge, T.T.: 1993, 'MAST: a mass spectrometer telescope for studies of the isotopic composition of solar, anomalous, and galactic cosmic rays', IEEE Trans. Geosci. Rem. Sensing 31, 557-564.

Cummings, A.C. and Stone, E.: 1988, 'Composition, gradients, and temporal variations of the anomalous cosmic-ray component', Proc. $6^{t h}$ Int. Solar Wind Conf. (Boulder) 306, 599-614.

Cummings, A.C., Mewaldt, R.A., Blake, J.B., Cummings, J.R., Fränz, M., Hovestadt, D., Klecker, B., Mason, G.M., Mazur, J.E., Stone, E.C., von Rosenvinge, T.T. and Webber, W.R.: 1995, 'Anomalous cosmic ray oxygen gradients throughout the heliosphere', Geophys. Res. Lett. 22, 341-344. 
Cummings, A.C. and Stone, E.C.: 1996, 'Composition of anomalous cosmic rays and implications for the heliosphere', Space Sci. Rev, 78, 117-128.

Cummings A.C. and Stone, E.C.: 1998, Space Sci. Rev, this volume.

Decker, R.B., Krimigis, S.M. and Kane, M.: 1995, 'Spatial gradients, energy spectra, and anisotropies of ions $\gtrsim 30 \mathrm{keV}$ at CIR shocks from 1 to $50 \mathrm{AU}$ ', Proc. $24^{\text {th }}$ Int. Cosmic Ray' Conf. (Rome) 4, $421-424$.

Earl, J.A.: 1974, 'A diffusive idealization of charged-particle transport in random magnetic fields', Astrophys. J. 193, 231-242.

Ellison, D.C., Jones, F.C. and Eichler, D.: 1981, 'Monte Carlo simulation of collisionless shocks showing preferential acceleration of high A/Z particles', J. Geophys. Res. 50, 110-113.

Ellison, D.C., Baring, M.G. and Jones, F.C.: 1996, 'Nonlinear particle acceleration in oblique shocks', Astrophys. J. 473, 1029-1050.

Fisk, L.A., Kozlovsky, B. and Ramaty, R.: 1974, 'An interpretation of the observed oxygen and nitrogen enhancements in low-energy cosmic rays', Astrophys. J. Lett. 190, L35-L37.

Geiss, J., Gloeckler, G. and von Steiger, R.: 1994, 'Solar and heliospheric processes from solar wind composition measurements', Phil. Trans. Roy. Soc. London A 349, 213-226.

Geiss, J., Gloeckler, G., Mall, U., von Steiger, R., Galvin, A.B. and Ogilvie, K.W.: 1994, 'Interstellar oxygen, nitrogen, and neon in the heliosphere', Astr. Astrophys. 282, 924-933.

Geiss, J., Gloeckler, G., Fisk, L.A. and von Steiger, R.: 1995, ' $\mathrm{C}^{+}$pickup ions in the heliosphere and their origin', J. Geophys. Res. 100, 23,373-23,377.

Geiss, J., Gloeckler, G. and von Steiger, R.: 1996, 'Origin of $C^{+}$ions in the heliosphere', Space Sci. Rev. 78, 43-52.

Giacalone, J. and Jokipii, J.R.: 1996, 'Perpendicular transport in shock acceleration', J. Geophys. Res. 101, 11,095-11,105.

Giacalone, J. and Jokipii, J.R.: 1997, 'Spatial variation of accelerated pickup ions at co-rotating interaction regions', submitted to Geophys. Res. Lett.

Giacalone, J., Jokipii, J.R., Decker, R.B., Krimigis, S.M., Scholer, M. and Kucharek, H.: 1997, 'The pre-acceleration of anomalous cosmic rays in the inner heliosphere', Astrophys. J. 486, 471-476.

Isenberg, P.A.: 1987, 'Evolution of interstellar pickup ions in the solar wind', J. Geophys. Res. 92, 1067-1073.

Jokipii, J.R.: 1987, 'Rate of energy gain and maximum energy in diffusive shock acceleration', Astrophys. J. 313, 842-846.

Jokipii, J.R.: 1991, in G.P. Zank and T.K. Gaisser (ed.), 'Diffusive shock acceleration: acceleration rate, magnetic-field direction and the diffusive limit', Particle Acceleration in Cosmic Plasmas, Am. Inst. of Phys., College Park, Md., 137-147.

Jokipii, J.R.: 1992, 'Constraints on the acceleration of anomalous cosmic rays', Astrophys. J. Lett. 393, L4 I-L43.

Jokipii, J.R., Kóta, J., Giacalone, J., Horbury, T.S. and Smith, E.J.: 1995, 'Interpretation and consequences of large-scale magnetic variances observed at high heliographic latitude', Geophys. Res. Lett. 22, 3385-3388.

Jokipii, J.R.: 1996, 'Theory of multiply-charged anomalous cosmic rays', Astrophys. J. Lett. 466, L47-L50.

Jokipii, J.R. and Giacalone, J.: 1998, Space Sci Rev., this volume.

Klecker, B., Hovestadt, D., Scholer, M., Arbinger, H., Ertl, M., Kästle, H., Künneth, E., Laeverenz, P., Seidenschwang, E., Blake, J.B., Katz, N. and Mabry, D.: 1993, 'HILT: a heavy ion large area proportional counter telescope for solar and anomalous cosmic rays', IEEE Trans. Geosci. Rem. Sensing 31, 542-548.

Klecker, B.: 1995, 'The anomalous component of cosmic rays in the 3-D heliosphere', Space Sci. Rev. 72, 419-430.

Klecker, B., McNab, M.C., Blake, J.B., Hamilton, D.C., Hovestadt, D., Kästle, H., Looper, M.D., Mason, G.M., Mazur, J.E. and Scholer, M.: 1995, 'Charge states of anomalous cosmic-ray nitrogen, oxygen, and neon: SAMPEX observations', Astrophys. J. Lett. 442, L69-L72.

Klecker, B., Oetliker, M., Blake, J.B., Hovestadt, D., Mason, G.M., Mazur, J.E. and McNab, M.C.: 1997, 'Multiply charged anomalous cosmic ray N, O, and Ne: observations with HILT/SAMPEX', Proc. $25^{\text {th }}$ Int. Cosmic Ray Conf. (Durban) 2, 273-276. 
Le Roux, J.A., Potgieter, M.S. and Ptuskin, V.S.: 1996, 'A transport model for the diffusive shock acceleration of anomalous cosmic rays in the heliosphere', J. Geophys. Res. 101, 4791-4803.

Lo, H.H., Kurzweg, L., Brackman, R.T. and Fite, W.L.: 1971, 'Electron capture and loss in collisions of heavy ions with atomic oxygen', Phys. Rev. A 4 (4), 1462-1478.

Luhn, A., Klecker, B., Hovestadt, D., Gloeckler, G., Ipavich, F.M., Scholer, M., Fan, C.Y. and Fisk, L.A.: 1984, 'Ionic charge states of $\mathrm{N}, \mathrm{Ne}, \mathrm{Mg}, \mathrm{Si}$, and $\mathrm{S}$ in solar energetic particle events', Adv. Space Res. 4, 161-164.

McDonald, F.B., Moraal, H., Reinecke, J.P.L., Lal, N. and McGuire, R.E.: 1992, 'The cosmic radiation in the heliosphere at successive solar minima', J. Geophys. Res. 97, 1557-1570.

McKibben, R.B., Connell, J.J., Lopate, C., Simpson, J.A. and Zhang, M.: 1996, 'Observations of galactic cosmic rays and the anomalous helium during Ulysses passage from the south to the north solar pole', Astron. Astrophys. 316, 547-554.

Mewaldt, R.A., Cummings, A.C., Cummings, J.R., Stone, E.C., Klecker, B., Hovestadt, D., Scholer, M., Mason, G.M., Mazur, J.E., Hamilton, D.C., von Rosenvinge, T.T. and Blake, J.B.: 1993, 'The return of the anomalous cosmic rays to $1 \mathrm{AU}$ in 1992', Geophys. Res. Lett. 20, 2263-2266.

Mewaldt, R.A., Cummings, J.R., Leske, R.A., Selesnick, R.S., Stone, E.C. and von Rosenvinge, T.T.: 1996a, 'A study of the composition and energy spectra of anomalous cosmic rays using the geomagnetic field', Geophys. Res. Lett. 23, 617-620.

Mewaldt, R.A., Selesnick, R.S., Cummings, J.R., Stone, E.C. and von Rosenvinge, T.T.: 1996b, 'Evidence for multiply charged anomalous cosmic rays', Astrophys. J. Lett. 466, L43-L46.

Miller, J.A. and Roberts, D.A.: 1995, 'Stochastic acceleration by cascading Alfvén waves in impulsive solar flares', Astrophys. J. 452, 912-932.

Oetliker, M., Klecker, B., Hovestadt, D., Mason, G.M., Mazur, J.E., Leske, R.A., Mewaldt, R.A., Blake, J.B. and Looper, M.D.: 1997a, 'The ionic charge of solar energetic particles with energies of 0.3-70 MeV per nucleon', Astrophys. J. 477, 495-501.

Oetliker, M., Klecker, B., Mason, G.M., McNab, M.C. and Blake, J.B.: 1997b, 'The abundance of anomalous cosmic ray carbon in the inner heliosphere', Proc. $25^{\text {th }}$ Int. Cosmic Ray Conf. (Durban) 2, 277-280.

Parker, E.N.: 1965 'The passage of energetic charged particles through interplanetary space', Planet. Space Sci. 13, 9-49.

Pesses, M.E., Jokipii, J.R. and Eichler, D.: 1981, 'Cosmic ray drift, shock-wave acceleration, and the anomalous component of cosmic rays', Astrophys. J. Lett. 246, L85-L88.

Reames, D.V., Barbier, L.M. and von Rosenvinge, T.T.: 1996, 'WIND/EPACT observations of anomalous cosmic rays,' Adv. Space. Res., in press.

Reinecke, J.P.L., Moraal, H. and McDonald, F.B.: 1993, 'The cosmic radiation in the heliosphere at successive solar minima: steady state no-drift solutions of the transport equation', J. Geophys. Res. 98, 9417-9431.

Reinecke, J.P.L., Moraal, H. and McDonald, F.B.: 1996, 'The cosmic radiation in the heliosphere at successive solar minima 3: steady state no-drift solutions of the transport equation', J. Geophys. Res. 101, 21,581-21,588.

Schlickeiser, R.: 1989, 'Cosmic-ray transport and acceleration. I. Derivation of the kinetic equation and application to cosmic rays in static cold media', Astrophys. J. 336, 243-263.

Selesnick, R.S., Cummings, A.C., Cummings, J.R., Mewaldt, R.A., Stone, E.C. and von Rosenvinge, T.T.: 1995, 'Geomagnetically trapped anomalous cosmic rays', J. Geophys. Res. 100, 9503-9518.

Selesnick, R.S., Mewaldt, R.A. and Cummings, J.R.: 1997, 'Multiply charged anomalous cosmic rays above $15 \mathrm{MeV}$ /nucleon', Proc. $25^{\text {th }}$ Int. Cosmic Ray Conf. (Durban) 2, 269-272.

Shafer, C.M., Gloeckler, G., Galvin, A.B., Ipavich, F.M., Geiss, J., von Steiger, R. and Ogilvie, K.: 1.993, 'Sulfur abundances in the solar wind measured by SWICS on ULYSSES', Adv. Space Res. 13 (6), 79-82.

Simnett, G.M. and Roelof, E.C.: 1998, Space Sci Rev, this volume.

Simpson, J.A.: 1995, 'The anomalous nuclear component in the three-dimensional heliosphere', Adv. Space Res. 16, 135-149.

Smith, E.J., Balogh, A.,-Neugebauer, M. and McComas, D.: 1995, 'Ulysses observations of Alfvén waves in the southem and northern hemispheres', Geophys. Res. Lett. 22, 3381-3384. 
Spjeldvik, W.: 1979, 'Expected charge states of energetic ions in the magnetosphere', Space Sci. Rev. 23, 499-538.

Steenberg, C.D. and Moraal, H.: 1996, 'An acceleration/modulation model for anomalous cosmic ray hydrogen in the heliosphere', Astrophys. J. 463, 776-783.

Steenberg, C.D. and Moraal, H.: 1997, 'An alternative approach to the modeling of anomalous cosmic ray intensities in the heliosphere', Adv. Space Res., accepted for publication.

Steenkamp, R. and Moraal, H.: 1993, 'Acceleration and modulation of anomalous helium and oxygen in 1977 and 1987', Proc. 23 ${ }^{\text {rd }}$ Int. Cosmic Ray Conf. (Calgary) 3, 419-422.

Steenkamp, R.: 1995, 'Shock acceleration as source of the anomalous component of cosmic rays in the heliosphere', Ph.D. Thesis, Potchefstroom University for CHE, Potchefstroom, South Africa.

Stone, E.C., Cummings, A.C. and Webber, W.R.: 1996, 'The distance to the solar wind termination shock in 1993 and 1994 from observations of anomalous cosmic rays', J. Geophys. Res. 101, 11,017-11,025.

Stone, E.C. and Cummings, A.C.: 1997, 'Evidence for anomalous cosmic ray S, Si, and Fe in the outer heliosphere and for a non-ACR source of S at 1 AU', Proc. $25^{\text {th }}$ Int. Cosmic Ray Conf. (Durban) $2,289-292$.

Takashima, T., Doke, T., Hayashi, T., Kikuchi, J., Kobayashi, M., Shirai, H., Takehana, N., Ehara, M., Yamada, Y., Yanagita, S., Hasebe, N., Kashiwagi, T., Kato, C., Munakata, K., Kohno, T., Kondoh, K., Murakami, H., Nakamoto, A., Yanagimachi, T., Reames, D.V. and von Rosenvinge, T.T.: 1997, 'The first observation of sulfur in anomalous cosmic rays by the GEOTAIL and the WIND spacecrafts', Astrophys. J. Lett. 477, L111-L113.

Tappin, S.J. and Simnett, G.M.: 1996, 'Modulation of anomalous cosmic ray ions by the interplanetary medium', Astrophys. J. 469, 402-411.

Trattner, K.J., Marsden, R.G., Bothmer, V., Sanderson, T.R., Wenzel, K.-P., Klecker, B. and Hovestadt, D.: 1996, 'Ulysses COSPIN/LET: latitudinal gradients of anomalous cosmic ray O, $\mathrm{N}$ and $\mathrm{Ne}^{2}$, Astron. Astrophys. 316, 519-527.

Vasyliunas, V.M. and Siscoe, G.L.: 1976, 'On the flux and the energy spectrum of interstellar ions in the solar system', J. Geophys. Res. 81, 1247-1252.

Verma, M.K., Roberts, D.A., Goldstein, M.L., Ghosh, S. and Stribling, W.T.: 1996, 'A numerical study of the nonlinear cascade of energy in magnetohydrodynamic turbulence', J. Geophys. Res. $101,21,619-21,625$.

Witte, M., Rosenbauer, H., Banaszkiewicz, M. and Fahr, H.: 1993, 'The Ulysses neutral gas experiment: determination of the velocity and temperature of the interstellar neutral helium', Adv. Space Res. 13, 121-130.

Zhou, Y. and Matthaeus, W.H.: 1990, 'Models of inertial range spectra of interplanetary magnetohydrodynamic turbulence', J. Geophys. Res. 95, 14,881-14,892.

Address for correspondence: Max-Planck-Institut für extraterrestrische Physik, 85740 Garching, Germany 